\title{
Event-triggered transmission for linear control over communication channels
}

\author{
Fulvio Forni ${ }^{\mathrm{a}, 1}$ Sergio Galeani ${ }^{\mathrm{b}, 2}$ Dragan Nešićc $^{\mathrm{c}, 3}$ Luca Zaccarian $^{\mathrm{d}, \mathrm{e}, 4}$ \\ ${ }^{a}$ Department of Electrical Engineering and Computer Science, Université de Liège, 4000 Liège, Belgium. \\ ${ }^{\mathrm{b}}$ DICII, University of Roma, Tor Vergata, Via del Politecnico 1, 00133 Roma, Italy \\ ${ }^{\mathrm{c}}$ EEE Department, University of Melbourne, Australia. \\ ${ }^{\mathrm{d}}$ CNRS, LAAS, 7 avenue du colonel Roche, F-31400 Toulouse, France and Univ. de Toulouse, LAAS, F-31400 Toulouse, France \\ e Dipartimento di Ingegneria Industriale, University of Trento, Italy
}

\begin{abstract}
We consider an exponentially stable closed loop interconnection of a continuous linear plant and a continuous linear controller, and we study the problem of interconnecting the plant output to the controller input through a digital channel. We propose a family of "transmission-lazy" sensors whose goal is to transmit the measured plant output information as little as possible while preserving closed-loop stability. In particular, we propose two transmission policies, providing conditions on the transmission parameters. These guarantee global asymptotic stability when the plant state is available or when an estimate of the state is available (provided by a classical continuous linear observer). Moreover, under a specific condition, they guarantee global exponential stability.
\end{abstract}

Key words: event-triggered sampling, hybrid system, asymptotic stability, non-periodic sample and hold.

\section{Introduction}

In recent years, much attention has been devoted to the study of closed-loop control systems interconnected by a digital channel where the information transmission is triggered by specific event-triggered conditions. The interest in this class of control systems is motivated by the increased computational capability required by control and estimation algorithms in addition to the presence of emerging control applications wherein the actuators of a control system may be non-colocated with the sensing devices (e.g, drilling systems, remote handling systems) so that the plant output is collected by the controller via

\footnotetext{
1 This paper presents research results of the Belgian Network DYSCO (Dynamical Systems, Control, and Optimization), funded by the Interuniversity Attraction Poles Programme, initiated by the Belgian State, Science Policy Office. The scientific responsibility rests with its authors. Work supported by FNRS. fforni@ulg.ac. be

2 Work supported by ENEA-Euratom and MIUR. galeani@disp.uniroma2.it

3 Work supported by the Australian Research Council under the Future Fellowship. d.nesic@ee. unimelb.edu.au

4 Work supported by HYCON2 Network of Excellence "Highly-Complex and Networked Control Systems", grant agreement 257462 and by the ANR project LimICoS, contract number 12 BS03 00501.
}

a digital channel which may have some stringent bandwidth requirement. This class of systems is a very specific subclass of the much more general topic of networked control systems (see, e.g., the recent surveys $[27,12]$ and references therein). Indeed, while in general networked control systems, various subcomponents are spread over a wide territory or are technologically built in such a way that several subcomponents of the control system communicate over shared and low capacity digital channels, the study of event-triggered and self-triggered systems $[2,5,6,13,16,23,25,26,28]$ led to a significant amount of research results where the core problem under consideration is that of two nodes (the sensing node and the actuating one) communicating through a (low capacity) digital channel where the transmission policy is determined based on suitable Lyapunov-like conditions involving some (more or less coarse) measurement of the plant state.

A natural way to represent and suitably write the dynamics of this specific two-nodes configuration is to use the hybrid systems notation, namely a state-space description wherein the state flows according to some continuous-time rules and, at some specific times, called jump times, it jumps following some discrete-time jump rule. A framework for the representation of hybrid systems that has been recently proposed in $[11,8]$ allows for 
a quite natural description of these phenomena with useful Lyapunov like results that have been proven to apply to large classes of systems described using this framework (see, e.g., $[3,4]$ and the survey $[9]$ ). This framework was used in connection with networked control systems in $[5,16]$, and recently in $[17,18]$, where Lyapunov tools are used to model ISS properties of networked control systems and the MATI (maximum allowable transfer interval), to preserve asymptotic stability.

Here, we consider a closed-loop system that consists of a linear controller driving a linear plant to guarantee closed-loop asymptotic stability, as shown in Figure 1, and we break the continuity of the transmission of the measured plant output $y$ to the controller input $u$ by introducing the transmission-lazy sensors, devices which measure the output $y$ and decide whether or not sending this measurement to the controller input $u$ through a transmission channel, based on non-periodic Lyapunovbased policies. We call these sensors "transmission-lazy" to resemble the fact that their goal is to avoid transmitting too often, so as to keep the digital channel load small enough. We suppose that each sensor is able to perform some computation on the measured plant output and, possibly, on extra available signals. The ideal scenario where this approach is relevant corresponds to cases where due to some technological constraint, there is a transmission line between a location where all the sensors are installed and a second location where the actuators are placed with a transmission channel inbetween (see Figure 1).

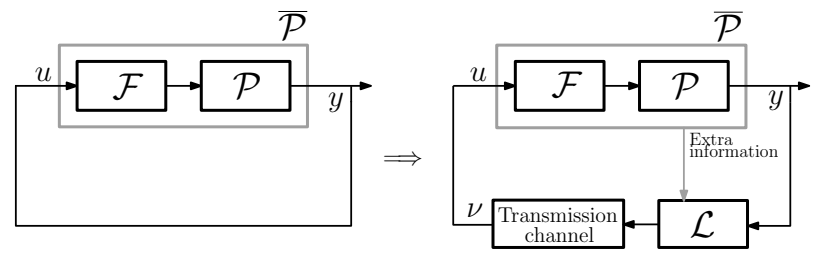

Figure 1. Nominal closed-loop $\mathcal{S}$ and transmission-lazy closed-loop system $\mathcal{S}_{\mathcal{L}}$.

The contribution of this paper consists in casting the above problem within the hybrid framework summarized in [9] and proposing two transmission policies for the transmission-lazy sensors which preserve the (global exponential) stability of the original closed-loop system. This result is achieved without requiring any modification to the design of the original controller. For simplicity, we first consider two transmission policies based on the state of the plant and the measurement error through a suitable Lyapunov-like function:

- a synchronous transmission policy where each sensor is aware of the conditions of the other sensors so that a transmission is a global decision of the sensing node. In this case, the sensors transmit a new sample all together when some suitable condition occurs;

- an asynchronous transmission policy where each sensor knows its own measurement error and the state of the plant, which is available in the sensing node. Then, it decides autonomously (namely, without any information on the measurement error of the other sensors) whether or not to transmit a new sample.

Then, we remove the dependence from the state by showing that the closed-loop results achieved by the transmission-lazy sensors are preserved when the information on the state (state-feedback) is replaced by an estimate from an observer (output feedback) located in the sensing node. To this aim, the adopted hybrid formulation is a fundamental tool.

Within the existing literature, the results in this paper can be seen as a specific application of the hybrid framework [9] to a peculiar control problem. In this sense, our paper is a constructive solution along the general lines of $[5,16,17]$, where Lyapunov tools and the hybrid framework of [9] are used in similar contexts. Moreover, the motivation behind our work is that of eventtriggered sampling where many interesting results have been published in recent years (see in $[6,13,23,25]$ and references therein). Additional work sharing the scenario of Figure 1 is that of $[22,15]$ and references therein, where the feedback signal is affected by an undesired quantization effect, rather than the presence of the communication channel. Within the event-triggered sampling context, taking into account linear systems, our work complements $[23,25]$, by casting similar problems and approaches within the hybrid systems framework, and proposing asynchronous transmission policies which are different from the synchronous ones considered in $[23,25]$. We show in the paper how restricting the attention to linear systems (whereas $[23,25]$ considers nonlinear systems) allows us to design transmission policies which lead to improved results, both in terms of architectures and of achievable performance, as compared to those in $[23,25]$ where, since a much more general nonlinear scenario is considered, the results obtained are more conservative. Finally, this work extends the results proposed in [7] by enforcing a dwell-time between transmissions and by introducing exponential bounds on the asymptotic stability guaranteed by the transmission policies. Finally, practical stability results of the output feedback case in [7] are now replaced by asymptotic (exponential) stability results.

The paper is structured as follows. In Section 2 we introduce the notation and give some preliminaries on hybrid systems. In Section 3 we introduce the problem data. Then, in Sections 4, 5, and 6 we illustrate the two policies first using information from the state of the plant, then relaxing this requirement by introducing an observer. Simulation examples are given in Section 8.

\section{Notation and preliminaries}

Given a vector $v, v^{T}$ denotes the transpose vector of $v$. Given two vectors $w$ and $v,\langle v, w\rangle=w^{T} v$. Given a set $a=$ $\left\{a_{1}, \ldots, a_{n}\right\}$ where $a_{i} \in \mathbb{R}$ for each $i=1 \ldots, n, \operatorname{diag}(a)$ denotes a diagonal matrix having the entries of $a$ on the 
main diagonal. Both the Euclidean norm of a vector and the corresponding induced matrix norm are denoted by $|\cdot|$. For a vector $v \in \mathbb{R}^{n}$ and a set $\mathcal{A} \subset \mathbb{R}^{n}|v|_{\mathcal{A}}:=$ $\inf _{y \in \mathcal{A}}|y-v|$. Given a set $\mathcal{A} \in \mathbb{R}^{n}$, the set $\mathcal{A}+\varepsilon \mathbb{B} \varepsilon \geq 0$, is the set of vectors $v$ such that $|v|_{\mathcal{A}} \leq \varepsilon$. A continuous function $\alpha: \mathbb{R}_{\geq 0} \rightarrow \mathbb{R}_{\geq 0}$ is said to belong to class $\mathcal{K}$ if it is strictly increasing and $\alpha(0)=0$; it belongs to class $\mathcal{K}_{\infty}$ if, moreover, $\lim _{r \rightarrow+\infty} \alpha(r)=+\infty$. For any $s \in \mathbb{R}$, consider the function $f: \mathbb{R} \rightarrow \mathbb{R}$ defined by $f(s)=0$ if $|s| \leq 1$, and $f(s)=\operatorname{sgn}(s)(|s|-1)$ if $|s| \geq 1$. Then, for any $s=\left[\begin{array}{lll}s_{1} & \ldots & s_{n}\end{array}\right]^{T} \in \mathbb{R}^{n}$, the deadzone function $\mathrm{dz}$ : $\mathbb{R}^{n} \rightarrow \mathbb{R}^{n}$ is given by $\mathrm{dz}(s)=\operatorname{diag}\left(f\left(s_{1}\right), \ldots, f\left(s_{n}\right)\right)$.

We summarize next the essential notation associated with the hybrid systems framework, outlined in [8], for which several results have been developed in $[11,19,20]$ and partially summarized in [9]. A hybrid system $\mathcal{H}$ is a tuple $(\mathcal{C}, \mathcal{D}, F, G)$, where $\mathcal{C} \subseteq \mathbb{R}^{n}$ and $\mathcal{D} \subseteq \mathbb{R}^{n}$ are, respectively, the flow set and the jump set, while $F: \mathbb{R}^{n} \rightrightarrows \mathbb{R}^{n}$ and $G: \mathbb{R}^{n} \rightrightarrows \mathbb{R}^{n}$ are set-valued mappings, called flow map and jump map, respectively. $F$ and $G$ characterize the continuous and the discrete evolution of the system, while $\mathcal{C}$ and $\mathcal{D}$ characterize subsets of $\mathbb{R}^{n}$ where such evolution may occur. A hybrid system is usually represented as follows

$$
\mathcal{H}=\left\{\begin{aligned}
\dot{x} \in F(x) & x \in \mathcal{C} \\
x^{+} \in G(x) & x \in \mathcal{D} .
\end{aligned}\right.
$$

Intuitively, the state continuously flows through $\mathcal{C}$, by following the dynamics given by $F$, or it jumps from $\mathcal{D}$, according to $G$. This hybrid evolution of the system can be conveniently characterized by using the notion of hybrid time domain which is a subset $E$ of $\mathbb{R}_{>0} \times \mathbb{N}$ given by the union of infinitely many intervals of the form $\left[t_{j}, t_{j+1}\right] \times\{j\}$ where $0=t_{0} \leq t_{1} \leq t_{2} \leq, \ldots$, or of finitely many such intervals, with the last one possibly of the form $\left[t_{j}, t_{j+1}\right] \times\{j\},\left[t_{j}, t_{j+1}\right) \times\{j\}$, or $\left[t_{j}, \infty\right] \times\{j\}$. Considering the notion of hybrid arc $x: \operatorname{dom} x \rightarrow \mathbb{R}^{n}$ given by (i) dom $x$ is a hybrid time domain and (ii) for each $j$, the function $t \mapsto x(t, j)$ is a locally absolutely continuous function on the interval $I_{j}=\{t:(t, j) \in \operatorname{dom} x\}$, we can define a solution to a hybrid system $\mathcal{H}$ as a hybrid arc which satisfies the following two conditions (i) for each $j \in \mathbb{N}$ such that $I_{j}$ has a nonempty interior

$$
\begin{array}{ll}
\dot{x}(t, j) \in F(x(t, j)) & \text { for almost all } t \in I_{j} \\
x(t, j) \in \mathcal{C} & \text { for all } t \in\left[\min I_{j}, \sup I_{j}\right),
\end{array}
$$

and (ii) for each $(t, j) \in \operatorname{dom} x$ such that $(t, j+1) \in$ $\operatorname{dom} x$,

$$
\begin{aligned}
x(t, j+1) & \in G(x(t, j)) \\
x(t, j) & \in \mathcal{D} .
\end{aligned}
$$

Solutions to hybrid systems may exist for a finite time, due to the constraints on the state motion enforced by the $\mathcal{C}$ and $\mathcal{D}$ sets. We say that a solution $x$ is maximal if there does not exists $x^{\prime}$ such that $x$ is a truncation of $x^{\prime}$ to some proper subset of dom $x^{\prime}$. We say that a solution $x$ is complete if $\operatorname{dom} x$ is unbounded.

Hybrid system $\mathcal{H}$ satisfies the basic conditions [9],[10] if - $\mathcal{C}$ and $\mathcal{D}$ are closed sets in $\mathbb{R}^{n}$;

- $F: \mathbb{R}^{n} \rightrightarrows \mathbb{R}^{n}$ is an outer semicontinuou 5 set-valued mapping, locally bounded on $\mathcal{C}$, and $F(x)$ is nonempty and convex for each $x \in \mathcal{C}$;

- $G: \mathbb{R}^{n} \rightrightarrows \mathbb{R}^{n}$ is an outer semicontinuous set-valued mapping, locally bounded on $\mathcal{D}$, and such that $G(x)$ is nonempty for each $x \in \mathcal{D}$.

These conditions are fundamental to guarantee robustness of the stability results presented in this paper.

Finally, following [9], for a hybrid system $\mathcal{H}$ the set $\mathcal{A}$ is (i) stable if for each $\epsilon>0$ there exists $\delta>0$ such that any solution $x$ to $\mathcal{H}$ with $|x(0,0)|_{\mathcal{A}} \leq \delta$ satisfies $|x(t, j)|_{\mathcal{A}} \leq \epsilon$ for all $(t, j) \in \operatorname{dom} x$; (ii) attractive if every maximal solution is complete and there exists $\delta>0$ such that any solution $x$ to $\mathcal{H}$ with $|x(0,0)|_{\mathcal{A}} \leq \delta$ is bounded and $|x(t, j)|_{\mathcal{A}} \rightarrow 0$ as $t+j \rightarrow \infty$, whenever $x$ is complete; (iii) asymptotically stable if it is both stable and attractive; (iv) exponentially stable if for some $\gamma>0$ and $k>0$, each solution $x$ to $\mathcal{H}$ satisfies $|x(t, j)|_{\mathcal{A}} \leq$ $k \exp (-\gamma(t+j))|x(0,0)|_{\mathcal{A}}$ for all $(t, j) \in \operatorname{dom} x$. For an asymptotically (exponentially) stable compact set $\mathcal{A}$, the basin of attraction $\mathcal{B}_{\mathcal{A}}$ is the set of points in $\mathbb{R}^{n}$ from which each solution is bounded and the complete solutions converge to $\mathcal{A}$. Finally, if $\mathcal{B}_{\mathcal{A}}=\mathbb{R}^{n}$ then $\mathcal{A}$ is globally asymptotically (exponentially) stable.

\section{The transmission-lazy closed-loop system}

Consider a nominal closed-loop system, $\mathcal{S}$, defined by the cascade interconnection $\overline{\mathcal{P}}$ of a linear controller $\mathcal{F}$ and a linear plant $\mathcal{P}$, given by

$$
\overline{\mathcal{P}}:\left\{\begin{array}{l}
\dot{x}=A x+B u \\
y=C x,
\end{array}\right.
$$

where $A, B, C$ are matrices, $x \in \mathbb{R}^{n}$, and $u, y \in \mathbb{R}^{q}$, and by the interconnection relation $u=y$ between the controller input and the plant measured output, from which we get

$$
\mathcal{S}:\left\{\begin{array}{l}
\dot{x}=(A+B C) x \\
y=C x
\end{array}\right.
$$

We consider the following standing assumption.

\footnotetext{
5 We recall here that a set valued mapping is outer semicontinuous if its graph is a closed set. Note that for single valued functions $f: \mathbb{R}^{n} \rightarrow \mathbb{R}^{n}$, outer semicontinuity is equivalent to continuity.
} 
Assumption 1 The nominal closed-loop system $\mathcal{S}$ is exponentially stable.

Consider now the introduction of a new device $\mathcal{L}$, the transmission-lazy sensors, or t-lazy sensors within the feedback interconnection, as shown in Figure 1. These intelligent sensors monitor the measured output $y$ and decide autonomously when transmitting a new sample of the output, denoted by $\nu$, to the control input $u$ with the twofold goal of preserving the stability of the closedloop system while breaking the continuity on the transmission of the measured plant output. Looking at Figure 1 , the arising transmission-lazy closed-loop system $\mathcal{S}_{\mathcal{L}}$, namely the closed-loop system of (2) through the interconnection $u=\nu$, is a hybrid system which combines together the continuous dynamics of the plant-controller cascade $\overline{\mathcal{P}}$ and the discrete dynamics of the t-lazy sensors $\mathcal{L}$. Its continuous dynamics can be modeled by

$$
\left\{\begin{array}{l}
\dot{x}=A x+B \nu \\
\dot{\nu}=0
\end{array}\right.
$$

where $x$ takes into account the plant-controller cascade dynamics while $\nu \in \mathbb{R}^{q}$ denotes the state of the t-lazy sensors, (each element $\nu_{i}$ of $\nu$ is related to the $i$ th-sensor), which replaces the interconnection $u=y$ of $\mathcal{S}$ with $u=$ $\nu$, which is kept constant during flows by $\dot{\nu}=0$. The discrete dynamics is given by

$$
\left\{\begin{array}{l}
x^{+}=x \\
\nu^{+}=g(x, \nu, \tau),
\end{array}\right.
$$

where $x$ does not change during jumps while $\nu$ is updated to $g(x, \nu, \tau), g: \mathbb{R}^{n} \times \mathbb{R}^{q} \times \mathbb{R}^{p} \rightarrow \mathbb{R}^{q}$, whose definition is given next and represent a transmission $\left(\tau \in \mathbb{R}^{p}\right.$ is an external timer characterized below).

Finally, we equip the t-lazy sensors with a bounded timer to guarantee a non-zero dwell-time between updates, whose dynamics is given by

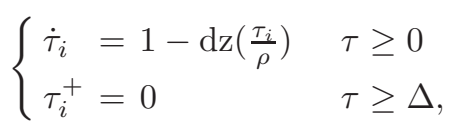

where $0<\Delta<\rho$ are design parameters, which guarantee that $\tau_{i}$ is bounded by $2 \rho$, it has rate 1 for $\tau_{i} \leq \rho$, and it may be reset to zero only if $\tau_{i} \geq \Delta$.

In what follows we will consider two scenarios in which either (i) one timer $\tau \in \mathbb{R}$ is shared among sensors, i.e. $p=1$ (synchronous policy), or (ii) each $i$ th sensor has its own timer $\tau_{i}$. i.e. $p=q$ (asynchronous policy). Thus, given $\left[\begin{array}{lll}\tau_{1} & \ldots & \tau_{p}\end{array}\right]^{T} \in \mathbb{R}^{p}, \overline{1}:=\left[\begin{array}{lll}1 & \ldots & 1\end{array}\right]^{T} \in \mathbb{R}^{p}$, and a function $h: \mathbb{R}^{n} \times \mathbb{R}^{q} \times \mathbb{R}^{p} \rightarrow \mathbb{R}^{p}$ which represents possible asynchronous resets of timers, the hybrid dynamics of the transmission-lazy closed-loop system (or t-lazy closed loop) can be summarized as follows:

$$
\begin{aligned}
\left\{\begin{array}{l}
\dot{x}=A x+B \nu \\
\dot{\nu}=0 \\
\dot{\tau}=\overline{1}-\operatorname{dz}\left(\frac{\tau}{\rho}\right)
\end{array} \quad(x, \nu, \tau) \in \mathcal{C}_{\Delta}\right. \\
\mathcal{S}_{\mathcal{L}}:\left\{\begin{array}{l}
x^{+}=x \\
\nu^{+}=g(x, \nu, \tau) \\
\tau^{+}=h(x, \nu, \tau) \\
y=C x .
\end{array} \quad(x, \nu, \tau) \in \mathcal{D}_{\Delta}\right.
\end{aligned}
$$

Within the model proposed in (7), the sets $\mathcal{C}_{\Delta}$ and $\mathcal{D}_{\Delta}$ and the parameter $\Delta$ and $\rho$ will be designed to decide whether or not to update $\nu$. Therefore, a transmission policy is given by the tuple $\left(\mathcal{C}_{\Delta}, \mathcal{D}_{\Delta}, \Delta, \rho\right)$.

Remark 1 The transmission of a sample is modeled in (7) as an instantaneous reset of the value $\nu$, which will typically assign to $\nu$ the current value of the output $y$. The proposed sample transmission model is a rough abstraction of a (possibly convoluted) process where the overall dynamics of the transmission channel plays a fundamental role. For example, the model does not consider transmission delays, noise corruption of the samples, packet drop, and many other features of digital transmission. While these phenomena concur to the evaluation of the closed-loop performance, within a certain bounded magnitude, they will not affect the main stability results established below. In particular, relying on the robustness to small perturbations guaranteed by the hybrid framework [8], the stability of our closed loop still holds in the presence of (small) transmission noise and delays (see Section 7).

\section{State feedback: synchronous transmission}

\subsection{The error dynamics}

We consider a synchronous transmission policy in which the transmission of the samples is a global decision based on the knowledge of $x$ and $\nu$ and of a timer state shared among sensors, i.e. $p=1$. The whole t-lazy sensors state $\nu$ is updated at once, that is $\nu=g(x, \nu, \tau):=C x=y$. Moreover, at updates, the timer state is reset to zero, that is, $h(x, \nu, \tau)=0$.

For simplicity of the exposition we consider the coordinate transformation $(x, e):=(x, \nu-y)$, from which we 
can rewrite the t-lazy closed loop (7) as follows

$$
\left.\begin{array}{l}
\left\{\begin{array}{l}
\dot{x}=F_{11} x+F_{12} e \\
\dot{e}=F_{21} x+F_{22} e \\
\dot{\tau}=1-\operatorname{dz}\left(\frac{\tau}{\rho}\right)
\end{array}\right. \\
\left\{\begin{array}{l}
x^{+}=x \\
e^{+}=0 \\
\tau^{+}=0
\end{array} \quad(x, e) \in \overline{\mathcal{C}} \text { or } 0 \leq \tau \leq \Delta\right.
\end{array}\right\}
$$

where $F_{11}:=(A+B C)$ is Hurwitz by Assumption 1, $F_{12}:=B, F_{21}:=-C(A+B C), F_{22}:=-C B$, and the relation between flow sets and jump sets before and after the coordinate transformation is given by $\mathcal{C}_{\Delta}:=$

$$
\left\{(x, \nu, \tau) \in \mathbb{R}^{n} \times \mathbb{R}^{q} \times \mathbb{R} \mid(x, \nu-y) \in \overline{\mathcal{C}} \text { or } 0 \leq \tau \leq \Delta\right\},
$$

and by $\mathcal{D}_{\Delta}:=$

$$
\left\{(x, \nu, \tau) \in \mathbb{R}^{n} \times \mathbb{R}^{q} \times \mathbb{R} \mid(x, \nu-y) \in \overline{\mathcal{D}} \text { and } \tau \geq \Delta\right\} .
$$

In what follows we use $F:=\left[\begin{array}{ll}F_{11} & F_{12} \\ F_{21} & F_{22}\end{array}\right]$.

By coordinate transformation, the closed-loop state is now directly related to the error $e$ between the current value of the output $y$ and the actual value of the samples $\nu$ transmitted to the controller. In fact, the synchronous transmission policy will require the transmission of a new sample when a particular relation between state $x$ and error $e$ holds. This behavior is modeled by the definition of the sets $\overline{\mathcal{C}}$ and $\overline{\mathcal{D}}$. In particular, from (8), the typical behavior of the t-lazy closed loop is to transmit a new sample only if the pair $(x, e)$ satisfies the criterion $(x, e) \in \overline{\mathcal{D}}$ and if at least $\Delta$ units of time have elapsed from the last transmission.

\subsection{The synchronous transmission policy}

The synchronous transmission policy $(\overline{\mathcal{C}}, \overline{\mathcal{D}}, \Delta, \rho)$ is based on two main parameters $\gamma_{e}>0$ and $\gamma_{x}>0$.

- $\gamma_{e}>0$ guarantees a proportionality between the norm of the error $e$ and the current state $x$, to avoid an asymptotic growth to infinity of the error $e$ while $x$ remains bounded. The conditions on $\gamma_{e}>0$ for the stability of the closed loop are very mild: every $\gamma_{e}>0$ guarantees stability;

- $\gamma_{x}>0$ specifies a bound on the decay rate of a given Lyapunov function $V$. This bound is directly connected to the frequency of the sample transmissions, since a transmission is required when the desired decrease on the Lyapunov function is not guaranteed anymore. The connection between sample transmission and Lyapunov-based conditions is given by the sets $\overline{\mathcal{C}}$ and $\overline{\mathcal{D}}$.
The Lyapunov function $V$ is suitably defined within condition (S1) below, and it is used in condition (S2) below to characterize $\overline{\mathcal{C}}$ and $\overline{\mathcal{D}}$. Note that, by definition, $\overline{\mathcal{C}} \neq \emptyset$.

(S1) Take $\gamma_{x}>0, Q=Q^{T}>0$, and $V: \mathbb{R}^{n} \times \mathbb{R}^{q} \rightarrow \mathbb{R}_{\geq 0}$,

$$
V(x, e):=\frac{1}{2}\left[\begin{array}{l}
x \\
e
\end{array}\right]^{T}\left[\begin{array}{cc}
P_{1} & 0 \\
0 & P_{2}
\end{array}\right]\left[\begin{array}{l}
x \\
e
\end{array}\right]
$$

such that

- $P:=\left[\begin{array}{cc}P_{1} & 0 \\ 0 & P_{2}\end{array}\right]$ is symmetric and positive definite,

- $F_{11}^{T} P_{1}+P_{1} F_{11} \leq-Q$,

- $\gamma_{x} I<Q$.

(S2) For any $\gamma_{e}>0$, define

$$
\begin{aligned}
& \overline{\mathcal{C}}:=\left\{\left.(x, e)\left|\left\langle\nabla V(x, e), F\left[\begin{array}{l}
x \\
e
\end{array}\right]\right\rangle \leq-\gamma_{x}\right| x\right|^{2} \text { and }|e| \leq \gamma_{e}|x|\right\} \\
& \overline{\mathcal{D}}:=\left\{\left.(x, e)\left|\left\langle\nabla V(x, e), F\left[\begin{array}{l}
x \\
e
\end{array}\right]\right\rangle \geq-\gamma_{x}\right| x\right|^{2} \text { or }|e| \geq \gamma_{e}|x|\right\} .
\end{aligned}
$$

We can now state the main result of the section, whose proof is provided in Section 4.3.

Theorem 1 Under Assumption 1, consider a transmission policy $(\overline{\mathcal{C}}, \overline{\mathcal{D}}, \Delta, \rho)$ which satisfies (S1) and (S2). Then, there exists $\Delta>0$ and $\rho>\Delta$ (sufficiently small) such that the compact set

$$
\mathcal{A}:=\{0\} \times\{0\} \times[0,2 \rho] \subset \mathbb{R}^{n} \times \mathbb{R}^{q} \times \mathbb{R}
$$

is globally asymptotically stable for the t-lazy closed-loop system (8). Moreover, if B in (2) is full column rank, then $\mathcal{A}$ is globally exponentially stable.

The reader will notice that the asymptotic stability of the set $\mathcal{A}$ in (13) entails asymptotic stability of the equilibrium $x=0$, which is the exponentially stable equilibrium of the original closed loop system (by construction $\overline{\mathcal{C}} \cup \overline{\mathcal{D}}=\mathbb{R}^{n} \times \mathbb{R}^{q} \times \mathbb{R}_{\geq 0}$, therefore every solution to (8) is complete). Moreover, under the mild hypothesis of $B$ full column rank, the exponential stability of the original closed-loop system is preserved by the synchronous transmission policy.

Note that, under Assumption 1, it is straightforward to see that the inequalities in $(\mathbf{S} 1)$ are feasible. In fact, for any given $\gamma_{x}$, there exists a matrix $Q=Q^{T}>0$ such that $\gamma_{x} I<Q$. Then, by Assumption 1, there exists a matrix $P_{1}=P_{1}^{T}>0$ which satisfies the condition $F_{11}^{T} P_{1}+P_{1} F_{11} \leq-Q$. Note that the inequality $\gamma_{x} I<Q$ guarantees that $\overline{\mathcal{C}} \neq \emptyset$. To see this, take $e=0$, then $\left\langle\nabla V(x, e), F\left[\begin{array}{l}x \\ e\end{array}\right]\right\rangle \leq-x^{T} Q x<-\gamma_{x}|x|^{2}$

Since the average sample transmission frequency is connected to the decay rate of the function $V$, this frequency can be partially regulated by suitably choosing $P_{1}, P_{2}$, 
$\gamma_{x}$, and $\gamma_{e}$. In the typical scenario, the transmission of one sample resets the error $e$, which typically increases during the flow interval after the sample transmission, weighted by $P_{2}$. Then, possibly, the boundary of the set $\overline{\mathcal{C}}$ is reached and a new sample is transmitted. In particular, from the definition of $\overline{\mathcal{C}}$, for an initial condition $e=0$ and $x \neq 0$, smaller values for $P_{2}$ guarantee longer flow intervals. In fact, for smaller values of $P_{2},(x, e)$ satisfies $\left\langle\nabla V(x, e), F\left[\begin{array}{l}x \\ e\end{array}\right]\right\rangle>-\gamma_{x}|x|^{2}$ for larger values of $e$. In the limit, that is, for $P_{2}=0$ and $\gamma_{e}=\infty$ (i.e. no bound on $e$ ), e may grow unbounded and $\mathcal{A}$ is not necessarily stable. But this is forbidden by condition (S1).

Remark 2 [Comparison with selected literature] The synchronous transmission policy is closely related to the event-triggered control approaches $[1,2, ?, 23,25]$ (which consider a more general class of nonlinear control systems) and $[6,13]$, which propose transmission policies based on inequalities on the measured output and on the state of the plant. In particular, the transmission policy in [23, Section IV] is similar to our synchronous policy, as shown by [23, Equation (13)] which also highlights some differences between our approach and the one in [23]. Specifically, for any choice of the event-triggering conditions used in [23] (which are expressed simply as inequalities involving the norms $|x|$ and $|e|$ ), there always exist an equivalent choice of the sets $\overline{\mathcal{C}}$ and $\overline{\mathcal{D}}$ in (12) and of $\Delta$ in (6) (with $\Delta<\tau$ and $\tau$ given by [23, Corollary IV.1]) yielding exactly the same events; the converse is not true, in general, due to the restricted dependence from $|x|$ and $|e|$ (instead of $x, e)$ in [23]. See also the simulation results of Section 8.1 where we compare these two approaches. As for [?], it proposes two event-triggering strategies and generalizes the assumptions in $[1,2,23,25]$. In particular, the proposed strategies are based on the relaxation of the input-to-state stability assumptions on the underlying (not triggered) system to global asymptotic stability assumptions. Unfortunately, this generalizations do not provide improvements on the transmission rate for LTI systems since on LTI systems the two properties are equivalent. Both [6] and [13] focus on more transmission-related implementation issues, thus being less related to the basic stabilization problem considered here; in [6] the optimization of a stochastic performance index measuring the state variance is considered for a network of simple dynamical systems, whereas in [13] the issue of balancing energy consumption in different nodes is tackled. Finally, alternative approaches are simpler to implement but require more bandwidth, like [5], which guarantees transmissions to happen before the expiration of the maximum allowable delay compatible with stability preservation.

While the main novelty of this paper as compared to previous approaches is in the proposed asynchronous transmission policy, even in the synchronous case we provide some advantages and novelties. First, in general, global exponential stability is guaranteed with less conservative bounds with respect to the current literature (see e.g. the above discussion about [23]). Then, the formulation within the hybrid systems framework of $[9,10]$ automatically provides some levels of robustness which are guaranteed by the framework itself, as well as several analysis tools that make it easier to establish some relevant properties and additional results (see e.g. the output feedback results in Section 6). Finally, an additional novelty is the introduction of a timer (the state $\tau$ ) within the sensors; while simple to implement, such modification enforces a minimum interval between consecutive transmissions of samples, meanwhile preserving stability (more recently, the same idea was used in [?]).

\subsection{Proof of Theorem 1}

The proof technique is inspired by [9, Example 27].

From the assumptions of the theorem, we provide a Lyapunov function $W$ in (14), we show that $W$ is nonincreasing at each sample transmission (at jumps) in (15), and we show that $W$ is non-increasing during flows, by decomposing the analysis in two parts: (i) for $\tau \in$ $[0, \Delta]$, and (ii) for $\tau \geq \Delta$ and $(x, e) \in \overline{\mathcal{C}}$. Combining these results with the invariance principle for Hybrid systems in [9, Theorem 23] and [19], we prove global asymptotic stability. Finally, based on a recent result in [24, Theorem 2], we strengthen the asymptotic convergence to an exponential one, by using the hypothesis that $B$ is full column rank.

Lyapunov function: using $X$ for the aggregate state $\left.\overline{\left[x^{T} e^{T} \tau\right.}\right]^{T}$, consider the following Lyapunov function

$$
W(X)=x^{T} P_{1} x+\exp ((2 \rho-\tau) \lambda) e^{T} P_{2} e
$$

where $\lambda>0$ is selected later. Using the definitions $\underline{\alpha}:=$ $\lambda_{\min }(P)$ and $\bar{\alpha}:=\max \left\{\lambda_{\max }\left(P_{1}\right), \exp (2 \rho \lambda) \lambda_{\max }\left(\bar{P}_{2}\right)\right\}$ we get $\underline{\alpha}|X|_{\mathcal{A}}^{2} \leq W(X) \leq \bar{\alpha}|X|_{\mathcal{A}}^{2}$ (radially unbounded).

Lyapunov function at jumps: we have that

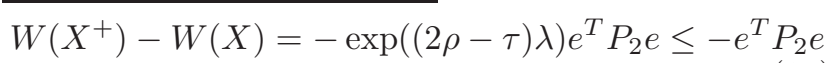

for each $X$ such that $(x, e) \in \overline{\mathcal{D}}$ and $\tau \geq \Delta$.

Lyapunov function on flows: the analysis is developed

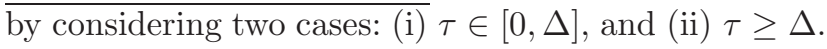

For (i), using $\varphi(\rho, \lambda, \tau):=\exp ((2 \rho-\tau) \lambda)$, considering $1-\mathrm{dz}\left(\frac{\tau}{\rho}\right)=1$, and defining $\lambda_{0}:=\lambda_{\min }(Q), \gamma_{1}:=$ $2\left|P_{1} F_{12}\right|, \gamma_{2}:=2\left|P_{2} F_{21}\right| \gamma_{3}:=2\left|P_{2} F_{22}\right|$, and $\gamma_{4}:=$ $\lambda_{\text {min }}\left(P_{2}\right)$, we get

$$
\begin{aligned}
\dot{W} \leq & -x^{T} Q x+2 x^{T} P_{1} F_{12} e \\
& +2 \varphi(\rho, \lambda, \tau) e^{T} P_{2}\left(F_{21} x+F_{22} e\right) \\
& -\lambda \varphi(\rho, \lambda, \tau) e^{T} P_{2} e \\
\leq & -\lambda_{0}|x|^{2}+\gamma_{1}|x||e|+\gamma_{2} \varphi(\rho, \lambda, \tau)|e||x| \\
& +\gamma_{3} \varphi(\rho, \lambda, \tau)|e|^{2}-\lambda \gamma_{4} \varphi(\rho, \lambda, \tau)|e|^{2} .
\end{aligned}
$$


Exploiting the inequality $a b \leq \frac{1}{\varepsilon} a^{2}+\varepsilon b^{2}$, where $\varepsilon>0$, and $a, b \in \mathbb{R}$, from (16) we get

$$
\begin{aligned}
\dot{W} \leq & -\lambda_{0}|x|^{2}+\gamma_{1} \frac{\varepsilon}{\varphi(\rho, \lambda, \tau)}|x|^{2}+\gamma_{1} \frac{\varphi(\rho, \lambda, \tau)}{\varepsilon}|e|^{2} \\
& +\gamma_{2} \varepsilon|x|^{2}+\gamma_{2} \frac{\varphi(\rho, \lambda, \tau)^{2}}{\varepsilon}|e|^{2} \\
& +\gamma_{3} \varphi(\rho, \lambda, \tau)|e|^{2}-\lambda \gamma_{4} \varphi(\rho, \lambda, \tau)|e|^{2} \\
\leq & \left(-\lambda_{0}+\gamma_{1} \varepsilon+\gamma_{2} \varepsilon\right)|x|^{2} \\
& +\varphi(\rho, \lambda, \tau) \underbrace{\frac{\gamma_{1}}{\varepsilon}+\frac{\gamma_{2} \varphi(\rho, \lambda, 0)}{\varepsilon}+\gamma_{3}-\lambda \gamma_{4}}_{=: \lambda_{1}})|e|^{2} \\
\leq & -\frac{1}{2} \lambda_{0}|x|^{2}-\varphi(\rho, \lambda, \tau) \lambda_{1}|e|^{2} \forall \tau \in[0, \Delta]
\end{aligned}
$$

where the first term of the last inequality follows from the selection of $\varepsilon:=\frac{\lambda_{0}}{2\left(\gamma_{1}+\gamma_{2}\right)}$, while $\lambda_{1}>0$ is achieved by picking $\lambda, \rho$ such that $\lambda>\frac{\gamma_{3}}{\gamma_{4}}+\frac{\gamma_{1}}{\gamma_{4} \varepsilon}+\frac{\gamma_{2} \varphi(\rho, \lambda, 0)}{\gamma_{4} \varepsilon}$, which can always be satisfied by picking $\lambda>0$ sufficiently large and $\rho>\Delta>0$ sufficiently small (i.e. for the design parameter $\Delta$ sufficiently small). For instance, define $c_{1}:=\frac{\gamma_{3}}{\gamma_{4}}+\frac{\gamma_{1}}{\gamma_{4} \varepsilon}$ and $c_{2}:=\frac{\gamma_{2}}{\gamma_{4} \varepsilon}$, the inequality above reads $\lambda>c_{1}+c_{2} \varphi(\rho, \lambda, 0)$, which holds for $\lambda:=c_{1}+2 c_{2}$ and $\rho$ sufficiently small, since $\varphi(\rho, \lambda, 0) \rightarrow 1$ as $\rho \rightarrow 0$.

For (ii), $\tau>\Delta$ implies $(x, e) \in \overline{\mathcal{C}}$. Thus, considering $1-\mathrm{dz}\left(\frac{\tau}{\rho}\right) \geq 0$, and using $\varphi(\rho, \lambda, \tau):=\exp ((2 \rho-\tau) \lambda)$, we get

$$
\begin{aligned}
\dot{W} \leq & -x^{T}\left(F_{11}^{T} P_{1}+P_{1} F_{11}\right) x+2 x^{T} P_{1} F_{12} e \\
& +2 \varphi(\rho, \lambda, \tau) e^{T} P_{2}\left(F_{21} x+F_{22} e\right) \\
= & \left\langle\nabla V(x, e), F\left[\begin{array}{l}
x \\
e
\end{array}\right]\right\rangle \\
& +2(\varphi(\rho, \lambda, \tau)-1) e^{T} P_{2}\left(F_{21} x+F_{22} e\right) \\
\leq & -\gamma_{x}|x|^{2}+2(\varphi(\rho, \lambda, \tau)-1) e^{T} P_{2}\left(F_{21} x+F_{22} e\right) \\
\leq & -\gamma_{x}|x|^{2}+2(\varphi(\rho, \lambda, \tau)-1)\left(\gamma_{2}|e||x|+\gamma_{3}|e|^{2}\right) \\
\leq & -\gamma_{x}|x|^{2}+2(\varphi(\rho, \lambda, \Delta)-1)\left(\gamma_{2} \gamma_{e}|x|^{2}+\gamma_{3} \gamma_{e}^{2}|x|^{2}\right) \\
\leq & -\frac{\gamma_{x}}{2}|x|^{2}
\end{aligned}
$$

where the last inequality holds for $\rho>\Delta$ sufficiently small. For example, take $\rho=2 \Delta$, then $\varphi(\rho, \lambda, \Delta)=$ $\varphi(2 \Delta, \lambda, \Delta)=\exp (3 \Delta \lambda) \rightarrow 1$, as $\Delta \rightarrow 0$. As in (17), the decreasing of $W$ in (18) is achieved by selecting the design parameter $\Delta$ sufficiently small.

Both cases (i) and (ii) are then covered by considering $\rho \in\left(0, \min \left\{\frac{\ln \left(c_{3} / c_{2}\right)}{2\left(c_{1}+c_{3}\right)}, \frac{1}{2 \lambda} \log \left(\frac{\gamma_{x}}{4\left(\gamma_{2} \gamma_{e}+\gamma_{3} \gamma_{e}^{2}\right)}+1\right)+\frac{\Delta}{2}\right\}\right)$.

GAS of the set $\mathcal{A}$ by invariance principle: since the tlazy closed-loop system in (8) satisfies the basic conditions of [9] (see Section 2), from the inequalities above, following [9, Theorem 23] or [19] $\mathcal{A}$ is stable. Moreover, for any given $\mu>0$, consider the level curve given by $\ell(\mu)=\{X \mid W(X)=\mu\}$. Suppose now that $X(0,0) \in \ell(\mu) \cap \overline{\mathcal{D}}$ with $e(0,0) \neq 0$. Then, from $(15)$
$W(X)$ decreases. Thus, suppose $X(0,0) \in \ell(\mu) \cap \overline{\mathcal{D}}$ with $e(0,0)=0$. From the definition of $\overline{\mathcal{D}}$ in $(12)$, necessarily $x=0$, thus $W(X)=0$ (in fact, for $e=0$ and $x \neq 0, X \notin \overline{\mathcal{D}})$. During flows each solution $X$ such that $X(0,0) \in \ell(\mu) \cap \overline{\mathcal{C}}$ and $x(0,0) \neq 0$ guarantees that $W(X)$ decreases (by (17) and (18)). For $X \in \ell(\mu) \cap \overline{\mathcal{C}}$ and $x(0,0)=0$, considering $\tau \leq \Delta$, we have that $W$ decreases (by (17)), while considering $\tau>\Delta$ we have $(x, e) \in \overline{\mathcal{C}}$ which implies $|e| \leq \gamma_{e}|x| \leq 0$, thus $W(X)=0$. Thus, using the fact that $\bar{W}(X)$ is radially unbounded and no complete solutions remain within $\ell(\mu)$, by $[9$, Theorem 23], the set $\mathcal{A}$ is globally asymptotically stable.

Exponential stability of the set $\mathcal{A}$ : it follows from the application of [24, Theorem 2]. For instance, decompose the state of the t-lazy closed-loop system in $\xi_{1}=(x, e)$ and $\xi_{2}=\tau$. Then, conditions 1)-3) of [24, Assumption 1] are satisfied. Moreover, $B$ full column rank implies the observability of the pair $\left(\left[\begin{array}{ll}I_{n} & 0\end{array}\right], F\right)$. In fact, using the linear transformation $T:=\left[\begin{array}{cc}I & 0 \\ -C & I\end{array}\right]$, we have $T^{-1}=\left[\begin{array}{ll}I & 0 \\ C & I\end{array}\right]$ from which $\left[\begin{array}{ll}A & B \\ 0 & 0\end{array}\right]=T^{-1} F T$ and $\left[\begin{array}{ll}I_{n} & 0\end{array}\right]=\left[\begin{array}{ll}I_{n} & 0\end{array}\right] T$. Thus, the observability of the pair $\left(\left[\begin{array}{ll}I_{n} & 0\end{array}\right], F\right)$ can be established via observability $\mathrm{PBH}$ test on the pair $\left(\left[\begin{array}{ll}I_{n} & 0\end{array}\right],\left[\begin{array}{cc}A & B \\ 0 & 0\end{array}\right]\right)$, that is,

$$
\operatorname{rank}\left[\begin{array}{c|c}
A-s I & B \\
\hline 0 & -s I \\
\hline I_{n} & 0
\end{array}\right]=n+q \quad \forall s \in \mathbb{C},
$$

which holds when $B$ is full column rank. Therefore, combining the observability of $\left(\left[I_{n} 0\right], F\right)$ with $(15)-(18)$ and with the bound on $W$ given after (14), condition 4) of [24, Assumption 1] is satisfied. Finally, the jumps of the t-lazy closed-loop system satisfy an average dwelltime constraint, since for each solution $X$ we have that $(t, j) \in \operatorname{dom} X$ implies $j \leq \frac{t}{\Delta}$, which satisfies condition $5)$ of [24, Assumption 1]. Thus, from [24, Theorem 2], $\mathcal{A}$ is globally exponentially stable.

Remark 3 The use of the timer $\tau$ guarantees a minimum dwell time $\Delta>0$ between transmissions. The proof of Theorem 1 provides a conservative bound on $\Delta$, which leads to excessively small values for $\Delta$, as revealed by the simulations. Relaxations on the bounds on $\Delta$ are possible but we will not pursue this analysis here. Note that there is a particular initial conditions from which a transmission may occur every $\Delta$ times, like, for example, $x=0$ and $e=0$. But such a transmission is only apparent, since $e^{+}=e=0$, thus the new transmission may be neglected.

\section{State feedback: asynchronous transmission}

\subsection{The error dynamics}

We consider an asynchronous transmission policy in which each sensor autonomously decides whether or not 
to transmit a new sample, based on its own state $\nu_{i}$, the timer $\tau_{i}$, and the state $x$ (which is assumed to be available to all sensors). We call asynchronous such a policy to underline the fact that the sensors transmit their measurements at independent times, and at different rates. The decision of a sample transmission is autonomous for each sensor in the precise sense that the single sensor does not need to know the error of any other sensor to decide its transmission. However, it uses the information on the state of the plant. A block diagram representing this scheme is shown in Figure 2.

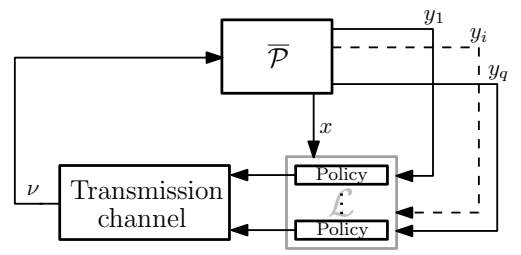

Figure 2. The transmission-lazy closed-loop system under the asynchronous transmission policy.

Consider the system in (7) with each sensor equipped with its own timer: $p=q$ and $\tau \in \mathbb{R}^{p}$. As in the previous section, the asynchronous transmission depends on Lyapunov-like conditions used to construct the sets $\mathcal{C}_{i}, \mathcal{D}_{i} \subseteq \mathbb{R}^{n} \times \mathbb{R} \times \mathbb{R}, i \in\{1, \ldots, q\}$. Each pair $\mathcal{C}_{i}, \mathcal{D}_{i}$ specifies when the $i$ th sensor may transmit a new sample.

To allow for an asynchronous update of the measurement vector, we define the functions $g$ and $h$ as follows

$g(x, \nu, \tau):=\left[\begin{array}{c}\eta_{1} \\ \vdots \\ \eta_{q}\end{array}\right]$ where $\begin{cases}\eta_{i}=(C x)_{i} & \text { if }\left(x, \nu_{i}, \tau_{i}\right) \in \mathcal{D}_{i} \\ \eta_{i}=\nu_{i} & \text { otherwise, }\end{cases}$

$h(x, \nu, \tau):=\left[\begin{array}{c}\eta_{1} \\ \vdots \\ \eta_{q}\end{array}\right]$ where $\begin{cases}\eta_{i}=0 & \text { if }\left(x, \nu_{i}, \tau_{i}\right) \in \mathcal{D}_{i} \\ \eta_{i}=\tau_{i} & \text { otherwise, }\end{cases}$

where $(C x)_{i}, \nu_{i}, \tau_{i}$ are the $i$ th elements of the vectors $C x, \nu, \tau$, respectively. Finally, the asynchronous transmission model can be completed by defining the sets $\mathcal{C}_{\Delta}$ and $\mathcal{D}_{\Delta}$ in $(7)$ as follows

$$
\begin{aligned}
& \mathcal{C}_{\Delta}=\left\{(x, \nu, \tau) \mid \forall i\left(x, \nu_{i}, \tau_{i}\right) \in \mathcal{C}_{i}\right\} \\
& \mathcal{D}_{\Delta}=\left\{(x, \nu, \tau) \mid \exists i\left(x, \nu_{i}, \tau_{i}\right) \in \mathcal{D}_{i}\right\}
\end{aligned}
$$

Thus, the definition of an asynchronous transmission policy $\left(\mathcal{C}_{\Delta}, \mathcal{D}_{\Delta}, \Delta, \rho\right)$ is equivalent to the definition of $\left(\mathcal{C}_{1}, \ldots, \mathcal{C}_{q}, \mathcal{D}_{1}, \ldots, \mathcal{D}_{q}, \Delta, \rho\right)$.

The asynchronous transmission mechanism modeled by (20), (21) (22) can be easily understood by considering the following scenario. Suppose that $\left(x, \nu_{i}, \tau_{i}\right)$ belongs to $\mathcal{D}_{i}$ for some $i$, and $\left(x, \nu_{j}, \tau_{j}\right) \notin \mathcal{D}_{i}$ for $j \neq i$. Looking at $(22)$, the t-lazy closed-loop system $\mathcal{S}_{\mathcal{L}}$ may jump. Then, from the definition of $g$ and $h$ in (20), (21), only the $i$ th sample will be transmitted, since $\nu_{i}^{+}=y_{i}$, and $\tau_{i}^{+}=0$, while $\nu_{j}^{+}=\nu_{j}$ and $\tau_{j}^{+}=\tau_{j}$.

Following the approach of previous section, we present the asynchronous transmission policy by using the coordinate transformation $(x, e)=(x, \nu-y)$, from which (7) becomes

$$
\begin{aligned}
& \left\{\begin{array}{l}
\dot{x}=F_{11} x+F_{12} e \\
\dot{e}=F_{21} x+F_{22} e \\
\dot{\tau}=\overline{1}-\mathrm{dz}\left(\frac{\tau}{\rho}\right)
\end{array}\right. \\
& \left\{\begin{array}{l}
x^{+}=x \\
e^{+}=g(x, e+y, \tau)-y \quad \exists i\left(\left(x, e_{i}\right) \in \overline{\mathcal{D}}_{i} \text { and } \tau_{i} \geq \Delta\right) \\
\tau^{+}=h(x, e+y, \tau)
\end{array}\right. \\
& y=C x
\end{aligned}
$$

from which, for each $i$, the sets $\overline{\mathcal{C}}_{i}, \overline{\mathcal{D}}_{i}$ are connected to the sets $\mathcal{C}_{i}, \mathcal{D}_{i}$ by the following relation $\mathcal{C}_{i}:=$ $\left\{\left(x, \nu_{i}, \tau_{i}\right) \in \mathbb{R}^{n} \times \mathbb{R} \times \mathbb{R} \mid\left(x, \nu_{i}-y_{i}\right) \in \overline{\mathcal{C}}_{i}\right.$ or $\left.0 \leq \tau_{i} \leq \Delta\right\}$ and $\mathcal{D}_{i}:=\left\{\left(x, \nu_{i}, \tau_{i}\right) \in \mathbb{R}^{n} \times \mathbb{R} \times \mathbb{R} \mid\left(x, \nu_{i}-y_{i}\right) \in\right.$ $\overline{\mathcal{D}}_{i}$ or $\left.\tau_{i} \geq \Delta\right\}$

Summarizing, from (23) and from the definition of $\overline{\mathcal{C}}_{i}$ and $\overline{\mathcal{D}}_{i}$, if $\left(x_{i}, e_{i}\right) \in \overline{\mathcal{D}}_{i}$ and $\tau_{i} \geq \Delta$, then the jump map of (23) guarantees that $e_{i}^{+}=0$ and $\tau_{i}^{+}=0$, otherwise $e_{i}^{+}=e_{i}$ and $\tau_{i}^{+}=\tau_{i}$. Moreover, the intersample time between two consecutive resets of each sensor $i$ is greater than or equal to $\Delta$, since, for each sensor $i$, resets are enabled only if the internal timer $\tau_{i} \geq \Delta$.

Remark 4 The transmissions of two or more sensors at the same time is modeled by a sequence of two or more consecutive resets. This case may occur when two or more indices $i$ satisfy the existential quantifier in (22). In such a case the jump rule is given by the union of two or more update laws in (20), (21). This definition produces an outer semicontinuous set-valued jump map, thereby guaranteeing robustness (see [11]). We do not elaborate further on robustness in this section, postponing the analysis of the robustness of the proposed algorithms to Section 7.

\subsection{The asynchronous transmission policy}

The asynchronous transmission policy is based on three parameters, $\gamma_{x}>0, \epsilon \in\left[0, \frac{1}{p}\right]$ and $\alpha \in \mathbb{R}^{q}$.

- $\gamma_{x}$ is used to establish a bound on the decay rate of a suitable Lyapunov function. This parallels the role of $\gamma_{x}$ in the previous section.

- The constant $\epsilon \in\left[0, \frac{1}{p}\right]$ is a lower bound on the value of each element of the vector $\alpha$. The presence of a lower bound allows for the possibility of varying the gains 
$\alpha$ at runtime (for performance improvement) without losing closed-loop stability.

- Each element $\alpha_{i}$ of $\alpha$ satisfies the condition $\alpha_{i}>\varepsilon$. Moreover, $\sum_{i=1}^{q} \alpha_{i}=1$ and each $\alpha_{i}$ is a weight on the achievable decay rate associated to sensor $i$. For example, when $\alpha_{i}$ is small the $i$-th sensors transmits at higher frequency. Therefore, the ratio between different elements of the vector $\alpha$ is in direct relation to the transmission rate of each sensor.

The conditions on the Lyapunov function are given in (A1) below. Flow and jump sets are based on this Lyapunov function and are given in (A2). Based on these parameters, we formulate the asynchronous transmission policy as follows.

(A1) Take $\gamma_{x}>0, \epsilon \in\left[0, \frac{1}{q}\right], Q=Q^{T}>0$, and $V$ : $\mathbb{R}^{n} \times \mathbb{R}^{q} \rightarrow \mathbb{R}_{\geq 0}$,

$$
V(x, e):=\frac{1}{2}\left[\begin{array}{l}
x \\
e
\end{array}\right]^{T}\left[\begin{array}{cc}
P_{1} & 0 \\
0 & P_{2}
\end{array}\right]\left[\begin{array}{l}
x \\
e
\end{array}\right]
$$

such that $P_{1}=P_{1}^{T}>0, P_{2}=\operatorname{diag}\left(p_{1}, \ldots, p_{q}\right)>0$ and

- $F_{11}^{T} P_{1}+P_{1} F_{11} \leq-Q$,

- $\frac{\gamma_{x}}{\epsilon} I<Q$.

(A2) Define

$$
a:=2\left|P_{1} F_{12}\right|, \quad b:=2\left|F_{21}\right|, \quad c:=2\left|F_{22}\right|,
$$

and for each for each $i \in\{1, \ldots, q\}$, define $\overline{\mathcal{C}}_{i}$ and $\overline{\mathcal{D}}_{i}$ respectively as

$$
\begin{aligned}
& \left\{\left.\left(x, e_{i}\right)\left|-\alpha_{i} x^{T} Q x+\left(a+b p_{i}\right)\right| x|| e_{i}\left|+c p_{i} e_{i}^{2} \leq-\gamma_{x}\right| x\right|^{2}\right\} \\
& \left\{\left.\left(x, e_{i}\right)\left|-\alpha_{i} x^{T} Q x+\left(a+b p_{i}\right)\right| x|| e_{i}\left|+c p_{i} e_{i}^{2} \geq-\gamma_{x}\right| x\right|^{2}\right\} .
\end{aligned}
$$

We can now provide the main result of the section, whose proof is provided in Section 4.3.

Theorem 2 Under Assumption 1, consider a transmission policy $\left(\overline{\mathcal{C}}_{1}, \ldots, \overline{\mathcal{C}}_{q}, \overline{\mathcal{D}}_{1}, \ldots, \overline{\mathcal{D}}_{q}, \Delta, \rho\right)$ which satisfies (A1) and (A2). Then, if $\sum_{i=0}^{q} \alpha_{i}=1$ and each $\alpha_{i}>\epsilon$, there exists $0<\Delta<\rho$ sufficiently small such that the compact set

$$
\mathcal{A}:=\{0\} \times\{0\} \times[0,2 \rho]^{q} \subset \mathbb{R}^{n} \times \mathbb{R}^{q} \times \mathbb{R}^{q}
$$

is globally asymptotically stable for the t-lazy closed-loop system (23). Moreover, if B in (2) is full column rank, then $\mathcal{A}$ is globally exponentially stable.

Theorem 2 establishes asymptotic stability of the set $\mathcal{A}$ in the asynchronous case, paralleling the synchronous results of Theorem 1. Like in the synchronous case, all maximal solutions to (23) are complete. It is worth to note however that the asynchronous transmission policy does not subsume the synchronous transmission policy. To see this, consider the implementation of both approaches to a single input single output (SISO) system. In this scenario conditions (26) are more conservative than conditions (12) due to the presence of extra terms that may lead to a higher sample transmissions rate. In fact, (26) are designed to (conservatively) compensate for the presence of many t-lazy sensors, which are not present in the SISO case.

For $i=1, \ldots, q, p_{i}$ can be considered as a weight on the single sensor error, and the combination of $\alpha_{i}$ and $p_{i}$ can be used to increase the update-rate of one sensor with respect to the others. For example, considering each $p_{i}=1$, a greater $\alpha_{i}$ allows for a larger error bound on the $i$ th sensor, thus the update-rate of that sensor decreases. Note also that each $\alpha_{i}$ can be modified at runtime. As long as $\sum_{i=0}^{q} \alpha_{i}=1$ and each $\alpha_{i}>\varepsilon>0$, global asymptotic stability is preserved.

As shown in previous section, $\overline{\mathcal{C}}_{i} \neq \emptyset$. To see this, consider $e=0$, then $-\alpha_{i} x^{T} Q x \leq-\epsilon x^{T} Q x \leq \gamma_{x}|x|^{2}$, from the last condition in (A1). Looking at the definitions of $\overline{\mathcal{C}}_{i}$ and $\overline{\mathcal{D}}_{i}$, note also that the information on the full state vector $x$ can be replaced by $|x|$ and $\left|x^{T} Q x\right|$ only which reduces dramatically the quantity of information required by each sensor. Moreover, the result of Theorem 2 still holds if $x^{T} Q x$ in $(26)$ is replaced by $\lambda_{\min }(Q)|x|^{2}$ and in this case each t-lazy sensor may decide its transmission by using only $e_{i}$ and $|x|$. Clearly, higher transmission frequency may occur since conservativeness is introduced.

\section{Remark 5 [Comparison with selected literature]}

An asynchronous transmission policy for a closed-loop system defined by the interconnection of several linear systems can be found in [26], where a separated triggering condition for each system is provided and, under specific decoupling conditions, it guarantees the stability of the interconnected system. As compared to that approach, our asynchronous transmission policy does not require any decoupling condition at the cost of using an additional information on the state of the controllerplant cascade, which is shared among the sensors. This shared information is used to decide whether or not to transmit a sampled output measurement $y_{i}$, without requiring the transmission of the full output vector $y$. A complementary approach can be found in [14] where each sensor may decide to trigger a transmission of the whole vector $y$, based on its local error $e_{i}$ and its partial knowledge of the state vector $x$.

\subsection{Proof of Theorem 2}

The proof of the theorem follows the line of the proof of Theorem 1. For instance, we introduce a Lyapunov function and we show the nonincresing features of the function at jumps and during flows. Then, based on the 
established inequalities, we apply the invariant principle in [9, Theorem 23] to show global asymptotic stability of the set $\mathcal{A}$. Global exponential stability is then established by invoking [24, Theorem 2], under the mild assumption that the matrix $B$ is full-column rank.

Lyapunov function: using $X$ for the aggregate state $\left.\overline{\left[x^{T} e^{T} \tau\right.}\right]^{T}$, consider the Lyapunov function $W$ : $\mathbb{R}^{n} \times \mathbb{R}^{q} \times \mathbb{R} \rightarrow \mathbb{R}_{\geq 0}$ given by

$$
W(X)=x^{T} P_{1} x+\sum_{i=1}^{q} p_{i} \exp \left(\left(2 \rho-\tau_{i}\right) \lambda\right) e_{i}^{2}
$$

where $\lambda>0$. Then, using $\underline{\alpha}, \bar{\alpha}$ defined in the proof of Theorem 1, $\underline{\alpha}|X|_{\mathcal{A}}^{2} \leq W(X) \leq \bar{\alpha}|X|_{\mathcal{A}}^{2}$.

Lyapunov function at jumps: we have that

$$
W\left(X^{+}\right)-W(X)=-p_{i} \exp \left(\left(2 \rho-\tau_{i}\right) \lambda\right) e_{i}^{2}
$$

for each $\left(x, e_{i}\right) \in \overline{\mathcal{D}}_{i}$ and $\tau_{i} \geq \Delta_{i}$, and $i \in\{1, \ldots, q\}$.

Lyapunov function on flows: we first establish a convenient bound on the dynamics. Using $\varphi_{i}\left(\rho, \lambda, \tau_{i}\right):=$ $\exp \left(\left(2 \rho-\tau_{i}\right) \lambda\right)$, for $i \in\{1, \ldots, q\}$, and $\varphi(\rho, \lambda, \tau):=$ $\operatorname{diag}\left\{\exp \left(\left(2 \rho-\tau_{1}\right) \lambda\right), \ldots, \exp \left(\left(2 \rho-\tau_{q}\right) \lambda\right)\right\}$, the derivative of $W$ is bounded by

$$
\begin{aligned}
\dot{W} \leq & -x^{T} Q x+2 x^{T} P_{1} F_{12} e \\
& +2 e^{T} \varphi(\rho, \lambda, \tau) P_{2}\left(F_{21} x+F_{22} e\right) \\
& -\lambda \operatorname{diag}\left(1-\operatorname{dz}\left(\frac{\tau}{\rho}\right)\right) e^{T} \varphi(\rho, \lambda, \tau) P_{2} e^{T} \\
\leq & -x^{T} Q x+a|x||e|+\sum_{i=1}^{q} \varphi_{i}\left(\rho, \lambda, \tau_{i}\right) p_{i}\left|e_{i}\right|(b|x|+c|e|) \\
& -\lambda \sum_{i=1}^{q}\left(1-\operatorname{dz}\left(\frac{\tau_{i}}{\rho}\right)\right) \varphi_{i}\left(\rho, \lambda, \tau_{i}\right) p_{i}\left|e_{i}\right|^{2} \\
\leq & \sum_{i=1}^{q}-\alpha_{i} x^{T} Q x+\left(a+\varphi_{i}\left(\rho, \lambda, \tau_{i}\right) p_{i} b\right)|x|\left|e_{i}\right| \\
& +\varphi_{i}\left(\rho, \lambda, \tau_{i}\right) p_{i}\left(c-\lambda\left(1-\mathrm{dz}\left(\frac{\tau_{i}}{\rho}\right)\right)\right)\left|e_{i}\right|^{2} .
\end{aligned}
$$

Then, we develop the analysis of (30) by considering two cases. Using $\pi_{i}:=-\alpha_{i} x^{T} Q x+(a+$ $\left.\varphi_{i}\left(\rho, \lambda, \tau_{i}\right) p_{i} b\right)|x|\left|e_{i}\right|+\varphi_{i}\left(\rho, \lambda, \tau_{i}\right) p_{i}\left(c-\lambda\left(1-\mathrm{dz}\left(\frac{\tau_{i}}{\rho}\right)\right)\right)\left|e_{i}\right|^{2}$ to simplify the notation, for each $i$, let us consider two cases: $(i) \tau_{i} \in[0, \Delta]$ and $(i i) \tau_{i} \geq \Delta$.

For $(i)$, considering $1-\mathrm{dz}\left(\frac{\tau_{i}}{\rho}\right)=1$ and using the inequality $s_{1} s_{2} \leq \frac{1}{\varepsilon} s_{1}^{2}+\varepsilon s_{2}^{2} \forall s_{1}, s_{2} \in \mathbb{R}, \varepsilon>0$, we get

$$
\begin{aligned}
\pi_{i} \leq & -\epsilon x^{T} Q x+\left(a+\varphi_{i}\left(\rho, \lambda, \tau_{i}\right) p_{i} b\right)|x|\left|e_{i}\right| \\
& +p_{i} \varphi_{i}\left(\rho, \lambda, \tau_{i}\right)(c-\lambda)\left|e_{i}\right|^{2} \\
\leq & -\epsilon \frac{\lambda_{0}}{2}|x|^{2}-\lambda_{1} \varphi_{i}\left(\rho, \lambda, \tau_{i}\right)\left|e_{i}\right|^{2}
\end{aligned}
$$

where the last inequality holds for $\lambda_{0}=\lambda_{\min }(Q)$, and for some $\lambda, \rho, \lambda_{1}>0$ ( $\rho, \lambda_{1}$ sufficiently small $)$, by using an argument similar to (16) and (17). In fact, the righthand side of the first inequality in (31) is very similar to the right-hand side of the last inequality in (16).

For $(i i)$, since $\tau_{i} \geq \Delta$, we have that $\left(x, e_{i}\right)$ belongs to $\overline{\mathcal{C}}_{i}$. Thus, as a first step, we claim the existence of a bound $\left|e_{i}\right| \leq \gamma_{i}|x|$ for some $\gamma_{i}:=\frac{\varepsilon \lambda_{\min }(Q)}{c p_{i}}$, which follows from the definition of $\overline{\mathcal{C}}_{i}$ in (26), by

$$
\begin{aligned}
& -\alpha_{i} x^{T} Q x+\left(a+b p_{i}\right)|x|\left|e_{i}\right|+c p_{i} e_{i}^{2} \leq-\gamma_{x}|x|^{2} \\
& \Rightarrow \quad c p_{i} e_{i}^{2} \leq \lambda_{\max }(Q)|x|^{2} \\
& \Rightarrow \quad e_{i}^{2} \leq \frac{\lambda_{\max }(Q)}{c p_{i}}|x|^{2} .
\end{aligned}
$$

Then, as a second step, since $1-\mathrm{dz}\left(\frac{\tau_{i}}{\rho}\right) \geq 0$ for $\tau_{i} \geq \Delta$, using the definition of $\overline{\mathcal{C}}_{i}$, we get

$$
\begin{aligned}
\pi_{i} \leq & -\alpha_{i} x^{T} Q x+\left(a+\varphi_{i}\left(\rho, \lambda, \tau_{i}\right) p_{i} b\right)|x|\left|e_{i}\right| \\
& +\varphi_{i}\left(\rho, \lambda, \tau_{i}\right) p_{i} c\left|e_{i}\right|^{2} \\
= & -\alpha_{i} x^{T} Q x+\left(a+p_{i} b\right)|x|\left|e_{i}\right|+p_{i} c\left|e_{i}\right|^{2} \\
& +\left(\varphi_{i}\left(\rho, \lambda, \tau_{i}\right)-1\right) p_{i}\left(b|x|\left|e_{i}\right|+c\left|e_{i}\right|^{2}\right) \\
\leq & -\gamma_{x}|x|^{2}+\left(\varphi_{i}(\rho, \lambda, \Delta)-1\right) p_{i}\left(b \gamma_{i}+c \gamma_{i}^{2}\right)|x|^{2} \\
\leq & -\frac{\gamma_{x}}{2}|x|^{2}
\end{aligned}
$$

where the last inequality holds for $\rho>0$ sufficiently small, as shown in (18) for a similar setup.

Define now $\mathcal{I}_{\Delta}:=\left\{i \mid \tau_{i} \leq \Delta\right\} \subseteq\{1, \ldots, q\}$ and use $\left|\mathcal{I}_{\Delta}\right|$ to denote the number of elements of $\mathcal{I}_{\Delta}$. Then, for $0<\Delta<\rho$ and $\rho$ sufficiently small, from $(i),($ ii $)$ and (30) we get

$\dot{W}(X) \leq-q \min \left(\frac{\epsilon \lambda_{0}}{2}, \frac{\gamma_{x}}{2}\right)|x|^{2}-\sum_{i \in I_{\Delta}} \lambda_{1} \varphi_{i}(\rho, \lambda, \Delta)\left|e_{i}\right|^{2}$

$\forall(x, e, \tau)$ such that $\forall i\left(\left(x, e_{i}\right) \in \overline{\mathcal{C}}_{i}\right.$ or $\left.0 \leq \tau_{i} \leq \Delta\right)$.

GAS of the set $\mathcal{A}$ by invariance principle: using the fact that (23) satisfies the basic conditions of [9] (see Section 2), combining (29), (34), and the bounds on $W(X)$ defined after (28), stability follows from [9, Theorem 23]. To establish global asymptotic stability (GAS) we proceed as in the proof of Theorem 1 . For any given $\mu>0$, consider the level curve given by $\ell(\mu)=\{X \mid W(X)=$ $\mu\}$. Suppose now that $X(0,0) \in \ell(\mu)$. From $(34)$, each solution $X$ from $\tau(0,0) \leq \Delta$ or from $\left(x(0,0), e_{i}(0,0)\right) \in$ $\overline{\mathcal{C}_{i}}, x(0,0) \neq 0$ guarantees that $W$ decreases. Moreover, each solution from $\left(x(0,0), e_{i}(0,0)\right) \in \overline{\mathcal{C}_{i}}, x(0,0)=0$, $\tau>\Delta$ necessarily has $e(0,0)=0$, which follows from (32). Suppose now $X(0,0) \in \ell(\mu)$ and $\left(x, e_{i}\right) \in \overline{\mathcal{D}}_{i}$ for some $i$. From (29), each solution $X$ from $e_{i}(0,0) \neq 0$ guarantees that $W(X)$ decreases. If $e_{i}(0,0)=0$, from 
(A2), $\frac{\gamma_{x}}{\epsilon}<Q$, and the definition of $\overline{\mathcal{D}}_{i}$ in (26), necessarily $x=0$. Thus, using the fact that $W(X)$ is radially unbounded and no complete solutions remain within $\ell(\mu)$, by [9, Theorem 23], the set $\mathcal{A}$ is GAS.

Exponential stability of the set $\mathcal{A}$ : it can be established by following an argument similar to to the proof of Theorem 1. For instance, using $\xi_{1}=(x, e)$ and $\xi_{2}=\tau$, conditions 1)-3) of [24, Assumption 1] are satisfied. $B$ full column rank implies the observability of $\left(\left[\begin{array}{ll}I_{n} & 0\end{array}\right], F\right)$, which combined with (29), (34) and with the bound on $W$ given after (28), condition 4) of [24, Assumption 1] is satisfied. Finally, the jumps of the t-lazy closed-loop system satisfy an average dwell-time constraint, since for each solution $X$, we have that $(t, j) \in \operatorname{dom} X$ implies $j \leq q \frac{t}{\Delta}$, (each sensor may reset at most $\frac{t}{\Delta}$ times). Thus, from [24, Theorem 2], $\mathcal{A}$ is globally exponentially stable.

\section{Output feedback approach}

Both the transmission policies presented in previous sections depend on the information from the state of the sensors and the controller-plant cascade. In this section we relax this formulation, showing that the state of the controller-plant cascade can be replaced by an estimate, through a classical linear continuous-time observer. We make the following assumption

\section{Assumption 2 The pair $(A, C)$ in $(2)$ is detectable.}

Considering the transmission-lazy closed-loop system in (7), the introduction of an observer leads to the following formulation.

$$
\begin{aligned}
& \left\{\begin{array}{l}
\dot{x}=A x+B \nu \\
\dot{\hat{x}}=A \hat{x}+B \nu+L(y-C \hat{x}) \\
\dot{\nu}=0 \\
\dot{\tau}=\overline{1}-\operatorname{dz}\left(\frac{\tau}{\rho}\right)
\end{array} \quad(\hat{x}, \nu, \tau) \in \mathcal{C}_{\Delta}\right. \\
& \left\{\begin{array}{l}
x^{+}=x \\
\hat{x}^{+}=\hat{x} \\
\nu^{+}=g(\hat{x}, \nu, \tau) \\
\tau^{+}=h(\hat{x}, \nu, \tau)
\end{array} \quad(\hat{x}, \nu, \tau) \in \mathcal{D}_{\Delta}\right.
\end{aligned}
$$

where the flow dynamics is enriched by the observer dynamics with gain $L \in \mathbb{R}^{n \times q}$, and where $\hat{x}$ replaces $x$ within the functions $g$ and $h$. Thus, looking at the definition of flow and jump sets in (35), transmissions depend now on the state $\nu$ of the sensors and the estimate $\hat{x}$ of the controller-plant cascade state. The following stability results extend the result of Theorems 1, 2 to the output feedback case.
Theorem 3 Under Assumption 2, suppose that $(A+$ $L C)$ is a Hurwitz matrix.

1. Consider the synchronous transmission policy of Section 4 and suppose that the hypothesis of Theorem 1 are satisfied. Then there exists $0<\Delta<\rho$ (sufficiently small) such that the set

$$
\mathcal{A}:=\{0\} \times\{0\} \times\{0\} \times[0,2 \rho] \subset \mathbb{R}^{n} \times \mathbb{R}^{n} \times \mathbb{R}^{q} \times \mathbb{R}
$$

is globally asymptotically stable for the closed-loop system (35). Moreover, if B in (2) is full column rank, then $\mathcal{A}$ in (36) is globally exponentially stable.

2. Consider the asynchronous transmission policy of Section 5 and suppose that the hypothesis of Theorem 2 are satisfied. Then there exists $0<\Delta<\rho$ (sufficiently small) such that the set

$$
\mathcal{A}:=\{0\} \times\{0\} \times\{0\} \times[0,2 \rho]^{q} \subset \mathbb{R}^{n} \times \mathbb{R}^{n} \times \mathbb{R}^{q} \times \mathbb{R}^{q}
$$

is globally asymptotically stable for the closed-loop system (35). Moreover, if B in (2) is full column rank, then $\mathcal{A}$ in (37) is globally exponentially stable.

The key point of Theorem 3 is in showing that the transmission policies do not need any modification if we implement them by replacing the state of the plant/controller cascade by an estimate. The proof of this fact is greatly simplified by the adoption of the hybrid framework of $[9],[11]$.

From the definition of $g$ in Sections 4 and 5 and looking at the jump dynamics of (35), each transmission is now based on estimate $\hat{y}:=C \hat{x}$ which replaces the measured output $y=C x$ and enforces a decoupled structure of the t-lazy closed-loop system. For instance, the policies are now based on $\hat{x}$, through a comparison between the quantities $\nu-C \hat{x}$ and $\hat{x}$. Thus, for example, the jump dynamics of the synchronous policy is now given by $\nu^{+}=C \hat{x}$ which allows for a mismatch dynamics at jumps given by $\nu^{+}-C \hat{x}^{+}=C \hat{x}-C \hat{x}=0$, paralleling the jumps dynamics of the state-feedback case in which $\nu^{+}=y=C x$ guarantees that $\nu^{+}-C x^{+}=y-C x=0$. Following this approach, the transmission policies operate on the subsystem $(\hat{x}, \nu)$, whose state is available, and the stability of the whole closed-loop system follows from the convergence of $\hat{x}$ to $x$, which is guaranteed by Assumption 2 and by the average dwell-time between jumps enforced by the timers dynamics.

Proof of Theorem 3. We extend the argument of the proofs of Theorems 1 and 2 to the observer dynamics. The proof is divided in two parts: the first one concerns the analysis of the synchronous case, while the second one develops the analysis of the asynchronous case. For each case, we first consider a generalized error system. Then, we give a Lyapunov function and we show that along the solutions to the hybrid system it satisfies several inequalities on the jump and flow dynamics. These inequalities are used in combination to an invariance 
principle to show global asymptotic stability. Finally, we strengthen these results to exponential bounds by relying on the presence of a dwell-time.

Synch, error dynamics and Lyapunov function: using the coordinate transformation $(\hat{x}, e, \eta)=(\hat{x}, \nu-C \hat{x}, x-$ $\hat{x})$ and considering the synchronous transmission policy of Section 4, we can rewrite (35) as follows

$$
\begin{aligned}
& \left\{\begin{array}{l}
\dot{\hat{x}}=F_{11} \hat{x}+F_{12} e+L C \eta \\
\dot{e}=F_{21} \hat{x}+F_{22} e-C L C \eta \\
\dot{\eta}=(A-L C) \eta \\
\dot{\tau}=\overline{1}-\mathrm{dz}\left(\frac{\tau}{\rho}\right)
\end{array}\right. \\
& \left\{\begin{array}{l}
\left.\hat{x}^{+}=\hat{x}, e\right) \in \overline{\mathcal{C}} \text { or } 0 \leq \tau \leq \Delta \\
e^{+}=0 \\
\dot{\eta}^{+}=\eta \\
\tau^{+}=0
\end{array} \quad(\hat{x}, e) \in \overline{\mathcal{D}} \text { and } \tau \geq \Delta\right.
\end{aligned}
$$

Using the aggregate state $X:=\left[\hat{x}^{T}, e^{T}, \tau\right]^{T}$ and $Y:=$ $\left[\hat{x}^{T}, e^{T}, \tau, \eta\right]^{T}$, consider $W(X)$ defined in (14), and define $P_{o}=P_{o}^{T}>0$ such that $(A+L C)^{T} P_{o}+P_{o}(A+L C) \leq$ $-I$, from which we can define the Lyapunov function $V: \mathbb{R}^{n} \times \mathbb{R}^{q} \times \mathbb{R} \times \mathbb{R}^{n} \rightarrow \mathbb{R}_{\geq 0}$ given by

$$
V(Y):=W(X)+\gamma \eta^{T} P_{o} \eta
$$

where $\gamma>0$. From the definition of $V$, using the bounds on $W$, there exists $\underline{\alpha}, \bar{\alpha}>0$ such that $\underline{\alpha}|Y|_{\mathcal{A}}^{2} \leq V(Y) \leq$ $\bar{\alpha}|Y|_{\mathcal{A}}^{2}$.

Synch, Lyapunov function at jumps: using (16), we have

$$
V\left(Y^{+}\right)-V(Y) \leq-e^{T} P_{2} e
$$

for each $Y$ such that $(\hat{x}, e) \in \overline{\mathcal{D}}$ and $\tau \geq \Delta$.

Synch, Lyapunov function on flows: (i) for $0 \leq \tau \leq \Delta$, using (17) and $\lambda_{0}, \lambda_{1}$ given in the last inequality of (17), pick $\lambda$ and $\rho$ as in the proof of Theorem 1 and define $\gamma_{0}:=\frac{\lambda_{0}}{2}, \gamma_{1}:=\lambda_{1} \exp ((2 \rho-\Delta) \lambda), \gamma_{2}:=2\left|P_{1} L C\right|$, $\gamma_{3}:=2 \exp (2 \rho \lambda)\left|P_{2} C L C\right|$. Then, we get

$$
\begin{aligned}
\dot{V}(Y) \leq & -\gamma_{0}|\hat{x}|^{2}-\gamma_{1}|e|^{2}+2 \hat{x}^{T} P_{1} L C \eta \\
& -2 \exp ((2 \rho-\tau) \lambda) e^{T} P_{2} C L C \eta-\gamma|\eta|^{2} \\
\leq & -\gamma_{0}|\hat{x}|^{2}-\gamma_{1}|e|^{2}+\gamma_{2}|\hat{x}||\eta|+\gamma_{3}|e||\eta|-\gamma|\eta|^{2} \\
\leq & -\frac{\gamma_{0}}{2}|\hat{x}|^{2}-\frac{\gamma_{1}}{2}|\hat{e}|^{2}-\frac{\gamma}{2}|\eta|^{2}
\end{aligned}
$$

where the last inequality is established by using $\varepsilon a^{2}+$ $\frac{1}{\varepsilon} b^{2} \geq a b a, b \in \mathbb{R}_{\geq 0}, \varepsilon>0$, for $\gamma>0$ sufficiently large. (ii) For $\tau \geq \Delta,(\hat{x}, e) \in \overline{\mathcal{C}}$, using (18) with $\rho$ sufficiently small, we get

$$
\begin{aligned}
\dot{V}(Y) & \leq-\frac{\gamma_{x}}{2}|\hat{x}|^{2}+\gamma_{2}|\hat{x}||\eta|+\gamma_{3}|e||\eta|-\gamma|\eta|^{2} \\
& \leq-\frac{\gamma_{x}}{2}|\hat{x}|^{2}+\left(\gamma_{2}+\gamma_{3} \gamma_{e}\right)|\hat{x}||\eta|-\gamma|\eta|^{2} \\
& \leq-\frac{\gamma_{x}}{4}|\hat{x}|^{2}-\frac{\gamma}{2}|\eta|^{2}
\end{aligned}
$$

where, as before, the last inequality holds for $\gamma>0$ sufficiently large.

Synch, GAS of $\mathcal{A}$ by invariance principle: from the inequalities above we can establish global asymptotic stability of the set $\mathcal{A}$ following the argument of the proof of Theorem 1. For instance, define $\ell(\mu):=\{Z \mid W(Z)=$ $\mu\}$. For each solution $Y:=(\hat{x}, \eta, e, \tau)$ such that $Y(0,0) \in$ $\ell_{\mu}$, we have that (i) on flows, when $\hat{x}, \eta \neq 0, V$ decreases; (ii) on flows, when $\hat{x}=0$ and $\eta=0$, either $V$ decreases or $(\hat{x}, e) \in \overline{\mathcal{C}}$, from which $e=0$, that is, $V(Y)=0$; (iii) on jumps, $V$ does not increase but after each jump the system must flow for a $\Delta$ interval of time thus, necessarily, $\ell(\mu)$ is not an invariant set. global asymptotic stability follows from [9, Theorem 23].

Synch, exponential stability of the set $\mathcal{A}$ : exponential stability of the set $\mathcal{A}$ in (36) can be established by using $[24$, Theorem 2], as in the proof of Theorem 1. In fact, the pair

$$
\left(\left[\begin{array}{ccc}
I_{n} & 0 & 0 \\
0 & 0 & I_{n}
\end{array}\right],\left[\begin{array}{ccc}
F_{11} & F_{12} & L C \\
F_{21} & F_{22} & C L C \\
0 & 0 & A-L C
\end{array}\right]\right)
$$

is observable when $B$ is full column rank (by linear transformation and $\mathrm{PBH}$-test). Thus, decomposing the state in $\xi_{1}=(\hat{x}, e, \eta)$ and $\xi_{2}=\tau$, using (40)-(42), and observing that $(t, j) \in \operatorname{dom} X$ implies $j \leq \frac{t}{\Delta}$, every condition of [24, Assumption 1] is satisfied. Therefore, $\mathcal{A}$ is globally exponentially stable from [24, Theorem 2$]$.

Asynch, error dynamics: for the asynchronous transmission policy, (35) becomes

$$
\begin{aligned}
& \left\{\begin{array}{lrl}
\dot{\hat{x}} & =F_{11} \hat{x}+F_{12} e+L C \eta & \\
\dot{e}=F_{21} \hat{x}+F_{22} e-C L C \eta & \forall i\left(\left(\hat{x}, e_{i}\right) \in \overline{\mathcal{C}}_{i}\right. \\
\dot{\eta}=(A+L C) \eta & \text { or } \left.0 \leq \tau_{i} \leq \Delta\right) \\
\dot{\tau}=\overline{1}-\operatorname{dz}\left(\frac{\tau}{\rho}\right) &
\end{array}\right. \\
& \left\{\begin{array}{lr}
\hat{x}^{+}=\hat{x} & \\
e^{+}=g(\hat{x}, e+C \hat{x}, \tau)-C \hat{x} & \exists i\left(\left(\hat{x}, e_{i}\right) \in \overline{\mathcal{D}}_{i}\right. \\
\dot{\eta}^{+}=\eta & \text { and } \left.\tau_{i} \geq \Delta\right) \\
\tau^{+}=h(\hat{x}, e+C \hat{x}, \tau) &
\end{array}\right.
\end{aligned}
$$

where $g$ and $h$ are defined in (20) and (21).

Asynch, Lyapunov inequalities: using $V$ in (39). at jumps we get

$$
V\left(Y^{+}\right)-V(Y) \leq-p_{i}\left|e_{i}\right|^{2}
$$


while on flows, using (34) and (32) with an argument similar to the sequence of inequalities (41) and (42), we get the following inequality

$$
\dot{V}(Y) \leq-\gamma_{1}|\hat{x}|^{2}-\gamma_{2}|\eta|^{2}-\gamma_{3} \sum_{i \in \mathcal{I}_{\Delta}}\left|e_{i}\right|^{2}
$$

where $\mathcal{I}_{\Delta}:=\left\{i \mid \tau_{i} \leq \Delta\right\} \subseteq\{1, \ldots, q\}$, for some $\gamma_{1}, \gamma_{2}, \gamma_{3}>0$.

Asynch, global asymptotic and exponential stability: using inequalities (45) and (46), the definition of the sets $\overline{\mathcal{C}}_{i}$ and $\overline{\mathcal{D}}_{i}$, and the fact that for any given solution $Y$, if $(t, j) \in \operatorname{dom} Y$ then $j \leq \frac{q t}{\Delta}$, we can establish that the set $\ell(\mu)$ is not invariant for any given $\mu>0$, from which global asymptotic stability of the set $\mathcal{A}$ in (37) follows by $[9$, Theorem 23]. Finally, exponential stability follows from [24, Theorem 2]. In fact, conditions 1)-4) of [24, Assumption 1] can be established as shown above for the synchronous policy, while condition 5) of [24, Assumption 1] follows from the fact that $(t, j) \in \operatorname{dom} Y$ implies $j \leq \frac{q t}{\Delta}$, for any given solution $Y$.

\section{Robustness of the transmission policies}

A fundamental feature of the proposed hybrid model (7) or (35) is that asymptotic stability is robust. In fact, these two models satisfy the so-called basic conditions [9], recalled in Section 2, which guarantee several interesting regularity properties of the space of solutions to the hybrid system. This regularity is exploited to establish several robustness results for hybrid systems stability. Based on these results, we show in this section that the stability proven in Theorems 1, 2, and 3 is indeed robust. This is one of the main advantages of modeling the sampling transmission policies within the hybrid systems framework proposed of [11],[9].

We will not enter into the details of robustness theory for hybrid systems. The interested reader is referred to [10]. Instead, we will show how to characterize parameter perturbations, measurements noise, and transmission delays as a perturbed hybrid model $\mathcal{H}_{\delta}$ where $\delta$ represents a perturbation radius with respect to the original system $\mathcal{H}$. Then, for example, we may invoke $[9$, Theorem 17] to establish that the asymptotic stability enforced by the transmission policies without perturbation turns to practical stability when parameters perturbations, measurement noise, and delays are sufficiently small. We do not propose any formal statement here. We will keep the exposition at level of a discussion.

Robustness to measurement noise, parameter uncertainties and transmission delays can be characterized as shown in $[9$, p.57]. For simplicity of exposition, let us consider only the error model of the synchronous case (8). The analysis of the other cases (23), (38), (44) is very similar. As a first step, let us rewrite (8) in the following simplified form

$$
\begin{aligned}
& \left\{\begin{array}{lr}
\dot{x}=f_{1}(x, e) & (x, e) \in \overline{\mathcal{C}} \\
\dot{e}=f_{2}(x, e) & \text { or } 0 \leq \tau \leq \Delta \\
\dot{\tau}=f_{3}(\tau) & (x, e) \in \overline{\mathcal{D}}
\end{array}\right. \\
& \begin{cases}x^{+}=x & \text { and } \tau \geq \Delta \\
e^{+}=0 & \\
\tau^{+}=0 & \end{cases}
\end{aligned}
$$

where the definition of $f_{1}, f_{2}$ and $f_{3}$ is clear from a comparison to (8). Then, we can address different kinds of perturbations by considering the following perturbed hybrid model.

$$
\begin{aligned}
& \left\{\begin{array}{lc}
\dot{x} \in f_{1}(x, e)+\delta_{1} \mathbb{B} & \left(x+d_{2}, e+d_{3}\right) \in \overline{\mathcal{C}}+\delta_{4} \mathbb{B} \\
\dot{e} \in f_{2}(x, e)+\delta_{2} \mathbb{B} & \text { or } 0 \leq \tau \leq \Delta \\
\dot{\tau} \in f_{3}\left(\tau+d_{1}\right)+\delta_{3} \mathbb{B} & \\
e^{+}=0+d_{4} & \left(x+d_{5}, e+d_{6}\right) \in \overline{\mathcal{D}} . \\
\tau^{+}=0 & \text { and } \tau \geq \Delta
\end{array}\right.
\end{aligned}
$$

where $d_{i}, i \in\{1, \ldots, 6\}$ are disturbance signals, and $\delta_{i}>0$ are constants. Note that $f_{i}(x, e)+\delta_{i} \mathbb{B}$ is now a set-valued map for each $i \in\{1,2,3\}$.

We can identify the following three cases of interest.

- Consider $\delta_{1}=\delta_{2}=\delta_{3}=\delta_{4}=0$ and suppose that The signal $d_{1}, \ldots, d_{6}$ are typically small, thus bounded within a ball of radius $\delta_{0} \mathbb{B}, \delta_{0}>0$. They characterize possible measurement noise that perturbs the system. Respectively, $d_{1}$ enforces a drift on the internal timer of the t-lazy sensors, $d_{2}, d_{3}, d_{5}, d_{6}$ introduce a perturbation on the transmission decision, $e=0+d_{4}$, that is, $v=y+d_{4}$ corrupts the transmitted sample.

- Suppose $d_{i}=0$ for each $i \in\{1, \ldots, 6\}, \delta_{4}=0$, and $\delta_{i}>0$ for each $i \in\{1,2,3\} . f_{i}(x, e)+\delta_{i} \mathbb{B}$ is a (outer semicontinuous, convex and not-empty) set valued mapping of radius $\delta_{i}$, centered at $f_{i}(x, e)$. The use of set-valued mappings is a possible approach to the characterization of parameters uncertainty on the model.

- Suppose $d_{i}=0$ for each $i \in\{1, \ldots, 6\}, \delta_{i}=0$ for each $i \in\{1,2,3\}$, and $\delta_{4}>0$. Since the flow set is now larger, also the space of solutions of the perturbed system is larger than the space of solutions of the original hybrid model (8). The new solutions have an "excess" of flow which exactly characterizes transmission delays. In fact, the excess of flow represents a scenario in which a sample transmission should be performed but the system continues to flow for a given (small) amount of time.

Using the fact that the original model (8) satisfies the basic conditions (see Section 2), the continuity of the flow 
map in (8), and the fact that each signal $d_{i}$ is bounded by $\delta_{0} \mathbb{B}$, we invoke $[9$, Theorem 17] (as done in [9, p.57]) to establish that for any given compact set of initial conditions $\mathcal{K}$ and any $\varepsilon>0$, there exist sufficiently small values of $\delta_{i}>0, i \in\{0,1,2,3,4\}$, such that all the solutions to (47) asymptotically (and uniformly) converge to the set $\mathcal{A}+\varepsilon \mathbb{B}$.

It follows that the synchronous transmission policy is robust to (small) measurement noise, parameters uncertainty and transmission delays. This property corresponds to semiglobal practical stability, where practical refers to the fact that solutions converge to a neighborhood of $\mathcal{A}$ given by $\mathcal{A}+\varepsilon \mathbb{B}$, while semiglobal refers to the fact that the initial compact set $\mathcal{K}$ can be taken arbitrarily large. Note that similar arguments can be used to establish robustness of each policy presented in this paper.

Remark 6 While this section illustrates the robustness of our scheme for small disturbances and/or perturbations, it is also of interest to characterize some level of robustness in the presence of large seldom events, such as packet corruption or loss within the transmission network. This phenomenon can be captured by adding an extra state to (4), (5), which acts like an input matched disturbance to the right hand side of the first equation in (4) and remains constant between jumps. Robustness to this (non-small) disturbance can then be addressed by making assumptions on its persistency (for example one may use dwell-time or average dwell-time assumptions between packet losses, that is nonzero occurrences of this disturbance) and exploiting the strict decrease given by our Lyapunov functions to dominate the effect of this large but not persistent disturbance. Developing this analysis is beyond the scope of this paper.

\section{Simulation examples}

\subsection{State-feedback and output feedback}

Consider an unstable plant given by the transfer function $\frac{s+2}{(s+1)(s-3)}$, which can be stabilized by negative static output feedback, for example by using the gain $k=9$. The controller-plant cascade can be represented by the following state space equations

$$
\left\{\begin{array}{l}
\dot{x}=\left[\begin{array}{cc}
2 & 1.5 \\
2 & 0
\end{array}\right] x+\left[\begin{array}{c}
-18 \\
0
\end{array}\right] u_{c} \\
y_{p}=\left[\begin{array}{ll}
0.5 & 0.5
\end{array}\right] x
\end{array}\right.
$$

and the nominal closed-loop system is given by (49) through the interconnection $u_{c}=y_{p}$.

We consider here the synchronous case. For $P_{1}=$ $\left[\begin{array}{ll}0.091 & 0.067 \\ 0.067 & 0.573\end{array}\right], 0<\gamma_{x} \ll 1, \gamma_{e} \gg 1$, and $P_{2} \in\{0.1,10\}$, conditions (S1) of Section 4 are satisfied and the effect of the t-lazy sensors on the trajectories of the system in the state-feedback case is summarized in Figures 3 and 4 . In all the simulations the state $\nu$ is initialized at zero for simplicity. The top row of Figure 3 represents the time evolution of the synchronous policy ( $x$ and $\nu)$ with state information available and without corruption of the transmitted samples for $P_{2}=0.1$ (black curve) and $P_{2}=10$ (gray curve). Note that the solid curve, corresponding to a smaller value of $P_{2}$ results in a significantly reduced data rate, as compared to the other selection (gray) whose trace is almost coincident with the nominal closed-loop (thin red curve), namely the closed-loop with no transmission channel. The bottom row of Figure 3 shows the same simulations with the addition of a uniform random number between -0.1 and 0.1 added to each transmitted sample, representing transmission noise affecting the communication channel. Comparing the solid traces in the upper and bottom rows it appears that the channel noise negatively affects the transmission rate but, as indicated in Section 7, closed-loop (practical) stability is preserved.

Figure 4 proposes a comparison between the case $P_{2}=$ 0.1 (solid curves in all traces of Figures 3 and 4 ) and the state-feedback policy in [23]. To apply the results of [23] to this example, we consider $V=x^{T} P_{1} x$ with the same $P_{1}$ used by our algorithm (defined above) and, from (8) we have that $\dot{V} \leq-\alpha|x|^{2}+\gamma|x||e|$ where $\alpha=1$ and $\gamma=4.046$. Thus, following the notation in [23, Equation (8)], we enforce a transmission of a sample when $\gamma|e| \geq$ $\sigma \alpha|x|$, for $\sigma=0.9<1$ (which preserves the decrease of $V$ along the solutions). The plots clearly illustrate the reduced transmission rate of our approach as compared to the policy based on [23, Equation (8)]. In particular, when the initial condition is balanced $\left(x(0,0)=\left[\begin{array}{ll}1 & 1\end{array}\right]^{T}\right.$ in the top row of Figure 4), a reduced transmission rate is already visible. However, the greatest advantage is experienced with the unbalanced initial condition $x(0,0)=$ $\left[\begin{array}{ll}10 & 1\end{array}\right]^{T}$ of the bottom row. It should be also recalled that the reduced transmission rate that we achieve is due to the fact that we restrict our attention to linear control systems, while the work in [23] addresses nonlinear systems for which more conservative bounds need to be used in general.

Figure 5 represents the time evolution of the synchronous policy from the estimated state $\hat{x}$ and without any noise. The observer gain is $L=\left[\begin{array}{c}-14.77 \\ -6.68\end{array}\right]$. For a fixed value of $P_{2}=0.1$, the convergence to zero depends on the initial mismatch between $\hat{x}$ and $x$.

\subsection{Asynchronous policy with weight variations}

We consider the following unstable linear plant

$$
\left\{\begin{array}{l}
\dot{x}_{p}=\left[\begin{array}{ll}
1 & 1 \\
0 & 1
\end{array}\right] x_{p}+\left[\begin{array}{ll}
1 & 0 \\
0 & 1
\end{array}\right] u_{p} \\
y_{p}=\left[\begin{array}{ll}
1 & 0 \\
0 & 1
\end{array}\right] x_{p} .
\end{array}\right.
$$

which can be stabilized by the following LQR gains

$$
y_{c}=\left[\begin{array}{ll}
-2.1961 & -0.7545 \\
-0.7545 & -2.7146
\end{array}\right] u_{c}
$$



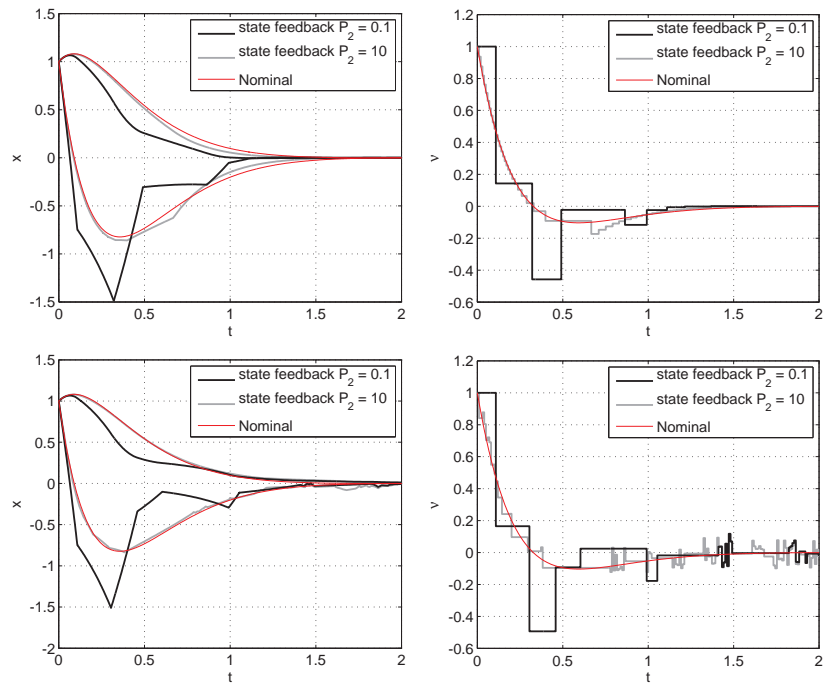

Figure 3. Synchronous policy from state feedback: time evolution of state $x$ and input $u_{c}$ for $P_{2} \in\{0.1,10\}$. TOP - no sample corruption. BOTTOM - sample corruption by uniform distributed noise between -0.1 and 0.1 .
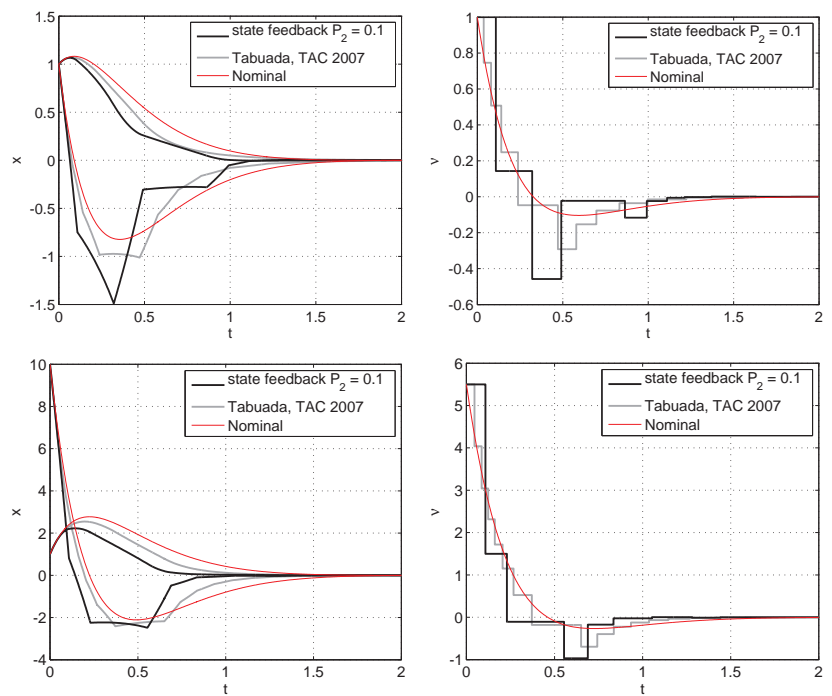

Figure 4. Comparison between the state-feedback synchronous policy for $P_{2}=0.1$ and the policy of [23]. TOP - comparison from the (balanced) initial condition $x(0,0)=\left[\begin{array}{ll}1 & 1\end{array}\right]^{T}$. BOTTOM - comparison from the (unbalanced) initial condition $x(0,0)=\left[\begin{array}{ll}10 & 1\end{array}\right]^{T}$.

through the interconnection $u_{p}=y_{c}$ and $u=y$. The effect of the introduction of the t-lazy sensors operating through the asynchronous policy is reported in Figure 6 . Note that by choosing different $\alpha_{1}$ and $\alpha_{2}$, we force one sensor to allow for a larger error bound on $e_{i}=\nu_{i}-y_{i}$ before transmitting, from which one sensor transmits its measurement $y_{i}$ more frequently than the other one.
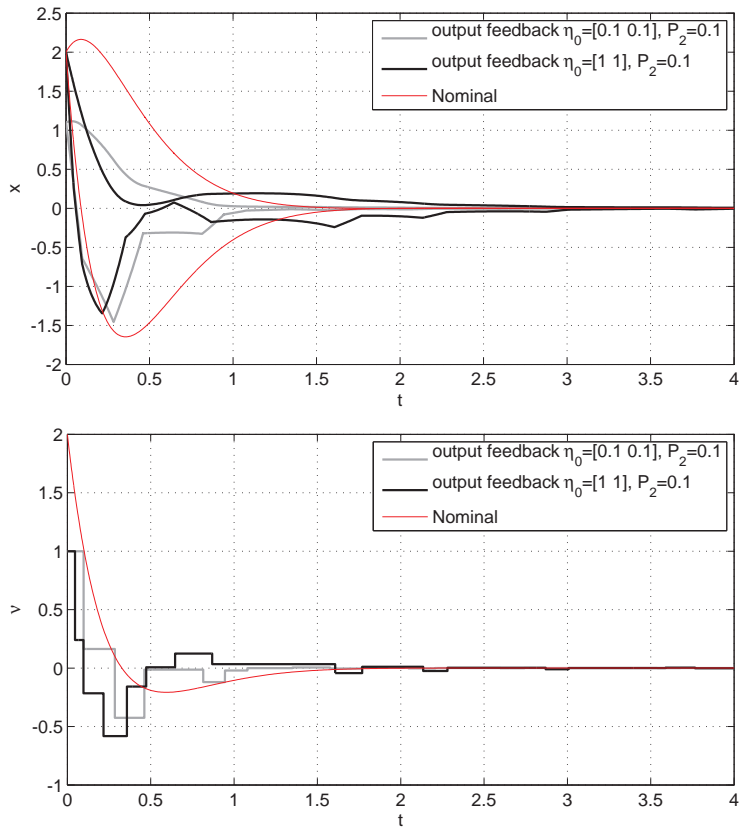

Figure 5. Synchronous policy from output feedback: time evolution of state $x$ and input $u_{c} . P_{2}=0.1$. Initial conditions: $x(0,0)=\left[\begin{array}{ll}1 & 1\end{array}\right]^{T}$, and $\eta(0,0)=\left[\begin{array}{ll}0.1 & 0.1\end{array}\right]^{T}$ - small estimation error (gray line), or $\eta(0,0)=\left[\begin{array}{ll}1 & 1\end{array}\right]^{T}$ - large estimation error (black line).

\section{Conclusions}

We introduced the transmission-lazy sensors to transform a continuous closed-loop system to a system whose feedback signal is sampled and transmitted, possibly over a digital channel, and we proposed two transmission policies which preserve the stability of the original closed-loop system. The first transmission policy requires an update of the whole measured output vector $y$ based on a centralized decision, while the second transmission policy allows for an asynchronous transmission, in which each sensor decides its own transmission. Moreover, when the input matrix is full column rank, we showed that these policies guarantee global exponential stability. Finally, by relying on an estimate of the state from a classical continuous-time observer, both approaches have been extended to the case in which only the output of the plant-controller cascade is available.

\section{References}

[1] A. Anta and P. Tabuada. Self-triggered stabilization of homogeneous control systems. American Control Conference, 2008. pages 4129-4134, 2008.

[2] A. Anta and P. Tabuada. To Sample or not to Sample: SelfTriggered Control for Nonlinear Systems. IEEE Transactions on Automatic Control, 55(9):2030-2042, 2010.

[3] C. Cai, A.R. Teel, and R. Goebel. Smooth Lyapunov functions for hybrid systems-Part I: Existence is equivalent to robustness. IEEE Transactions on Automatic Control, 52(7):1264-1277, 2007. 

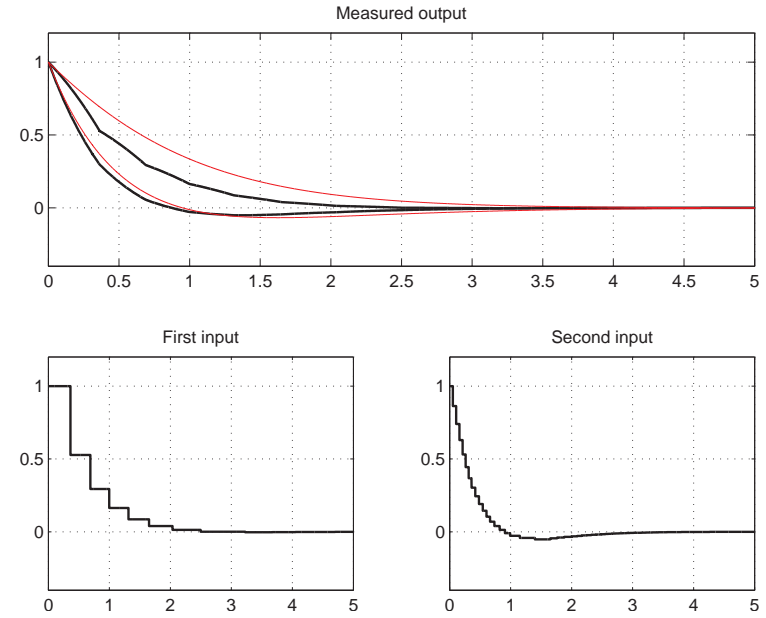

(a) $P_{2}=I, \alpha_{1}=0.9, \alpha_{2}=0.1$
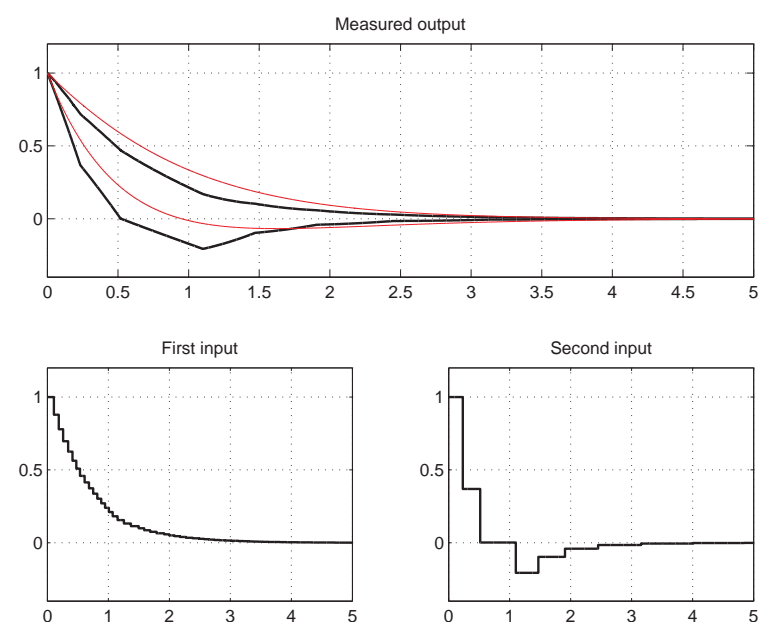

(b) $P_{2}=I, \alpha_{1}=0.1, \alpha_{2}=0.9$

Figure 6. Asynchronous policy. Effects of variations on the vector $\alpha$. The thin line in each figure is the output of the nominal closed-loop system.

[4] C. Cai, A.R. Teel, and R. Goebel. Smooth Lyapunov functions for hybrid systems Part II:(pre) asymptotically stable compact sets. IEEE Transactions on Automatic Control, 53(3):734-748, 2008.

[5] D. Carnevale, A.R. Teel, and D. Nešić. Further results on stability of networked control systems: a Lyapunov approach. In American Control Conference, 200\%. ACC '07, pages 1741 -1746 , July 2007.

[6] A. Cervin and T. Henningsson. Scheduling of event-triggered controllers on a shared network. In 47 th IEEE Conference on Decision and Control, 2008. CDC 2008., pages 3601-3606, December 2008.

[7] A. Forni, S. Galeani, D. Nešić, and L. Zaccarian. Lazy sensors for the scheduling of measurement samples transmission in linear closed loops over networks. In 49th IEEE Conference on Decision and Control, 2010. CDC 2010, pages 6469-6474, 2010.

[8] R. Goebel, J. Hespanha, A.R. Teel, C. Cai, and R.G. Sanfelice. Hybrid systems: Generalized solutions and robust stability. In NOLCOS, pages 1-12, Stuttgart, Germany, 2004.

[9] R. Goebel, R. Sanfelice, and A.R. Teel. Hybrid dynamical systems. Control Systems Magazine, IEEE, 29(2):28-93, April 2009.
[10] R. Goebel, R. Sanfelice, and A.R. Teel. Hybrid Dynamical Systems: modeling, stability, and robustness Princeton University Press, 2012.

[11] R. Goebel and A.R. Teel. Solutions to hybrid inclusions via set and graphical convergence with stability theory applications. Automatica, 42(4):573 - 587, 2006.

[12] JP Hespanha, P. Naghshtabrizi, and Y. Xu. A survey of recent results in networked control systems. Proceedings of the IEEE, 95(1):138-162, 2007.

[13] M. Mazo and P. Tabuada. On event-triggered and selftriggered control over sensor/actuator networks. In 47 th IEEE Conference on Decision and Control, 2008. CDC 2008., pages 435-440, December 2008.

[14] M. Mazo Jr., P. Tabuada. Decentralized event-triggered control over wireless sensor/actuator networks. IEEE Transactions on Automatic Control, Special Issue on Wireless Sensor and Actuator Networks: to appear. arXiv:1004.0477.

[15] D. Nesic and D. Liberzon. A unified framework for design and analysis of networked and quantized control systems. IEEE Transactions on Automatic Control, 54(4):732-747, april 2009.

[16] D. Nešić and A.R.Teel. Input output stability properties of networked control systems. IEEE Trans. Automat. Contr., 49:1650-1667, 2004.

[17] R. Postoyan, A. Anta, D. Nešić, and P. Tabuada. A unifying Lyapunov-based framework for the event-triggered control of nonlinear systems. In 50th IEEE Conference on Decision and Control and European Control Conference, pages 2559-2564, 2011.

[18] R. Postoyan, P. Tabuada, D. Nešić, and A. Anta. Eventtriggered and self-triggered stabilization of network control systems. In 50th IEEE Conference on Decision and Control and European Control Conference, 2011.

[19] R.G. Sanfelice, R. Goebel, and A.R. Teel. Invariance principles for hybrid systems with connections to detectability and asymptotic stability. IEEE Transactions on Automatic Control, 52(12):2282-2297, 2007.

[20] R.G. Sanfelice, R. Goebel, and A.R. Teel. Generalized solutions to hybrid dynamical systems. ESAIM: $C O C V$, 14(4):699-724, October 2008.

[21] A. Seuret and C. Prieur and N. Marchand Stability of nonlinear systems by means of event-triggered sampling algorithms. In IMA Journal of Mathematical Control and Information, 2013.

[22] Y. Sharon and D. Liberzon. Input to state stabilizing controller for systems with coarse quantization. IEEE Transactions on Automatic Control, 57(4):830-844, 2012.

[23] P. Tabuada. Event-triggered real-time scheduling of stabilizing control tasks. IEEE Transactions on Automatic Control, 52(9):1680-1685, September 2007.

[24] A.R. Teel, F. Forni, and L. Zaccarian. Lyapunov-based sufficient conditions for exponential stability in hybrid systems. IEEE Transactions on Automatic Control, 58(6):1591-1596, 2013.

[25] X. Wang and M.D. Lemmon. Event design in event-triggered feedback control systems. In 47th IEEE Conference on Decision and Control, 2008. CDC 2008., pages 2105-2110, December 2008.

[26] X. Wang and M.D. Lemmon. Event-triggered broadcasting across distributed networked control systems. In American Control Conference, 2008, pages 3139-3144, june 2008.

[27] TC Yang. Networked control system: a brief survey. IEE Proceedings-Control Theory and Applications, 153(4):403$412,2006$.

[28] W. Zhang and X. Xu. Analytical design and analysis of mismatched smith predictor. ISA Transaction, 40(2):133138, April 2001. 